\title{
Ribociclib Nanostructured Lipid Carrier Aimed for Breast Cancer: Formulation Optimization, Attenuating In Vitro Specification, and In Vivo Scrutinization
}

\author{
Ali Sartaj, ${ }^{1}$ Annu, ${ }^{1}$ Largee Biswas, ${ }^{2}$ Anita Kamra Verma, ${ }^{2}$ P. K. Sahoo, ${ }^{3}$ Sanjula Baboota $\left(\mathbb{D},{ }^{1}\right.$ \\ and Javed Ali $\mathbb{D}^{1}$ \\ ${ }^{1}$ Department of Pharmaceutics, School of Pharmaceutical Education and Research, Jamia Hamdard, New Delhi 110062, India \\ ${ }^{2}$ Nanobiotech Lab, Kirori Mal College, University of Delhi, Delhi 110007, India \\ ${ }^{3}$ Department of Pharmaceutics, Delhi Institute of Pharmaceutical Sciences and Research, New Delhi 110017, India \\ Correspondence should be addressed to Javed Ali; javedaali@yahoo.com
}

Received 21 September 2021; Revised 2 December 2021; Accepted 28 December 2021; Published 3 February 2022

Academic Editor: Sanyog Jain

Copyright (C) 2022 Ali Sartaj et al. This is an open access article distributed under the Creative Commons Attribution License, which permits unrestricted use, distribution, and reproduction in any medium, provided the original work is properly cited.

\begin{abstract}
Purpose. The current investigation is on the explicit development and evaluation of nanostructured lipidic carriers (NLCs) through the oral route to overcome the inherent lacuna of chemotherapeutic drug, in which Ribociclib (RBO) was used for breast cancer to diminish the bioavailability issue. Method. The RBO-NLCs were prepared using the solvent evaporation method and optimized method by the Box-Behnken design (BBD). Various assessment parameters characterized the optimized formulation and their in vivo study. Results. The prepared NLCs exhibited mean particle size of $114.23 \pm 2.75 \mathrm{~nm}$, mean polydispersity index of $0.649 \pm 0.043$, and high entrapment efficiency of $87.7 \pm 1.79 \%$. The structural analysis by TEM revealed the spherical size of NLCs and uniform drug distribution. An in vitro drug release study was established through the $0.1 \mathrm{~N} \mathrm{HCl}$ $\mathrm{pH} 1.2$, acetate buffer $\mathrm{pH} 4.5$, and phosphate buffer $\mathrm{pH} 6.8$ with \% cumulative drug release of $86.71 \pm 8.14,85.82 \pm 4.58$, and $70.98 \pm 5.69 \%$, was found respectively, compared with the RBO suspension (RBO-SUS). In vitro intestinal gut permeation studies unveiled a 1.95-fold gain in gut permeation by RBO-NLCs compared with RBO-SUS. In vitro lipolysis suggests the drug availability at the absorption site. In vitro haemolysis study suggests the compatibility of NLCs to red blood cells compared to the suspension of the pure drug. The confocal study revealed the depth of penetration of the drug into the intestine by RBO-NLCs which was enhanced compared to RBO-SUS. A cell line study was done in MCF-7 and significantly reduced the $\mathrm{IC}_{50}$ value compared to the pure drug. The in vivo parameters suggested the enhanced bioavailability by 3.54 times of RBO-NLCs as compared to RBO-SUS. Conclusion. The in vitro, ex vivo, and in vivo results showed a prominent potential for bioavailability enhancement of RBO and effective breast cancer therapy.
\end{abstract}

\section{Introduction}

The incidence of cancerous disease recently increased in recent years, impacting the physical, mental, and social life of humans. The incidence varies from developed countries (1 to $2 \%$ ) to fewer development countries (almost 5\%) yearly. The estimate also showed that more than 7 million people die from cancer disease and is predicted to be 10 to 15 million new cases added by 2020. Meanwhile, this disease is an unexceptional malignant breast cancer found in women, with more than 1 million fresh cases added yearly
[1]. Breast cancer was ranked as number 1 due to the number in Indian women with an age rate as high 25.8 per 100,000 and a mortality rate of 2.7 per 100,000 women [2]. There are lots of chemotherapy drugs available for breast cancer; however, Ribociclib (RBO) is one of them that has been recently approved by the US FDA, and it is given orally of $200 \mathrm{mg}$ as shown in Figure 1. The mechanism of action of $\mathrm{RBO}$ is to hamper the activity of cyclin-dependent kinase (CDK) at different types of cyclins like CDK4 and CDK6 of cell cycle pathways which indirectly inhibit the retinoblastoma $(\mathrm{Rb})$ protein phosphorylation. The hampering of $\mathrm{Rb}$ 


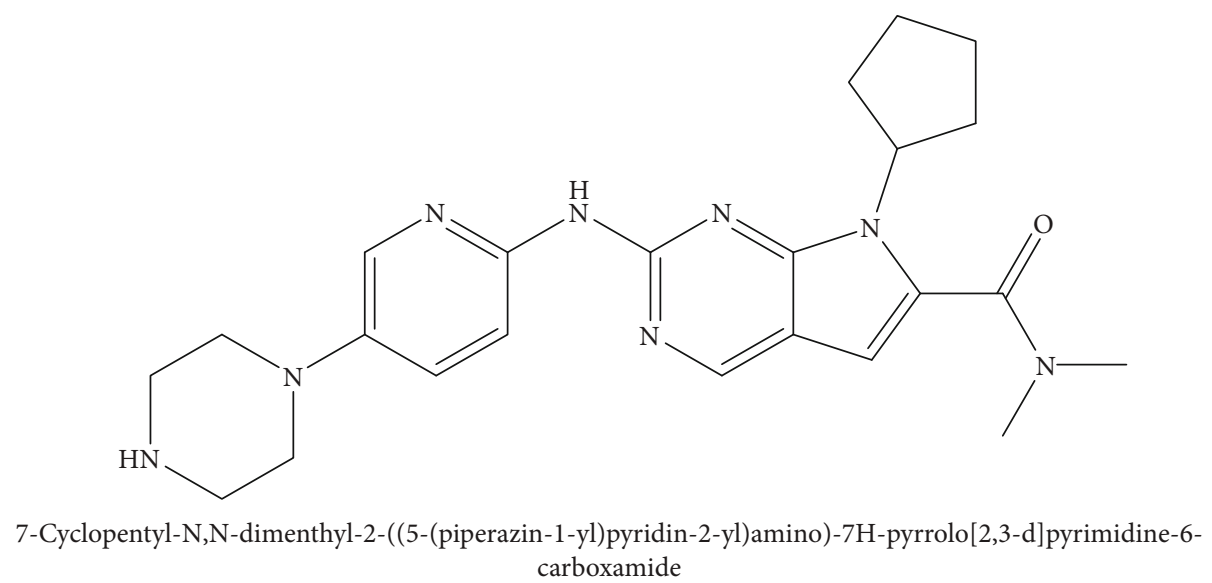

FIgUre 1: Chemical structure of Ribociclib with its IUPAC name.

again slows down the CDK-mediated G1-S phase movement. Because of this, the cell cycle is apprehended, inhibiting the cancer cell growth $[3,4]$. The RBO is associated with various adverse effects, and the most common reaction, with more than $20 \%$ incidence, is neutropenia, nausea, fatigue, diarrhea, leukopenia, alopecia, vomiting, constipation, headache, and back pain. Apart from adverse effect, the drug is also associated with low solubility and permeability as it is a BCS class IV drug and undergoes immense metabolism by hepatic cells mediated via CYP3A4 in the human liver. Ribociclib is also the substrate for P-gp efflux, which leads to an increase in the dose to achieve the therapeutic level [5]. Thus, these major factors which decrease the degree of absorption of Ribociclib lead to its poor in vivo prospects. The clinical efficacy of Ribociclib is not satisfactory at the therapeutic level, which often results in an increase in dose frequency, or high dose causes adverse effects [6]. However, being a recently approved drug (RBO), not much work has been done so far in the nanocarrier system to overcome the related issue. A recent study investigated by Fei and Yoosefian on the preparation of RBO micelles to increase the aqueous solubility of hydrophobic drug carried out using dodecylphosphocholine surfactant [7]. The remaining challenge still to overcome is the bioavailability issue by inhibiting P-gp efflux and bypassing the extensive first-pass metabolism [8]. The lipidic nanocarriers are being developed in order to overcome the predicaments like improving solubility, minimization of P-gp efflux, protection of the drug from enzymatic (CYP3A4) degradation, providing metabolic stability (stability in GIT), enhancing permeability and retention in the target tissue, enhancing the bioavailability, and minimization of toxic drug effects [9]. The drug integrated into nanostructured lipidic carrier (NLCs) formulations with specific lipid (Solid and Liquid lipids) and excipients can regulate P-gp-mediated efflux movement and has the potential to change the pharmacokinetics of Ribociclib largely. In addition, lipids used in Ribociclibloaded NLCs will promote its systemic blood circulation via the intestinal lymphatic system rather than being directly absorbed into the portal circulation and consequently pre- vent its significant first-pass metabolism [10]. Thus, our objective is to develop NLCs as a formulation which exhibits great potential for improving and normalizing the absorption of Ribociclib and contributing significantly in enhancing its therapeutic efficacy up to a satisfactory level.

\section{Materials}

RBO was obtained as gift samples from Alembic Pharmaceuticals Pvt. Ltd., Hyderabad, India. Various emulsifiers, oils, and lipids such as Labrafil M 1944 CS, Labrafil M 2125 CS, Maisine 351, Campritol 888 ATO, Precirol ATO 5, Gelot 64, Gelucire 44/14, Gelucire 44/01, and Solutol HS 15 were procured from Gattefosse India Pvt. Ltd. Capmul MCM, Capmul PG-12, Caprol PGE-860, were obtained from Abitec Corporation Cleveland, USA. Poloxamer 188, Cremophor EL and RH were obtained from BASF Mumbai, India. Tween 20 and Tween 80 were procured from Merck, Germany. Distilled deionized water used obtained from Milli-Q (Millipore, Bedford, MA, USA). The reagents used in all methods were of analytical grade available in the lab facility.

\section{Methods}

\subsection{Assessment of Excipients}

3.1.1. Evaluation and Selection of Liquid Lipids. The selection process for liquid lipid encompassed taking $2 \mathrm{ml}$ of liquid lipid in a glass vial to which a surplus quantity of drug was added to evaluate the maximum solubility. The glass vials containing lipid and drug were tightly stoppered and maintained on a mechanical shaker for $72 \mathrm{~h}$ at $25.0 \pm 0.5^{\circ} \mathrm{C}$ . This was further centrifuged at $5000 \mathrm{rcf}$, and the supernatant was dissolved in methanol before quantitatively analyzing using a UV-spectroscopy at a wavelength of $282 \mathrm{~nm}$ [11].

3.1.2. Evaluation and Selection of Solid Lipids. Similarly, for solid lipid selection, the known quantity, i.e., $100 \mathrm{mg}$ of the drug, was taken in a glass vial to which solid lipid was added up in surplus amount slowly until the solid lipid solubilizes 
the drug. The temperature was maintained at higher than the actual melting point $\left(5^{\circ} \mathrm{C}\right.$ above the melting point) of each solid lipid. The amount wherein an unclouded solution has been seen was taken as the endpoint [11].

3.1.3. Compatibility of Solid and Liquid Lipids. To determine the solid and liquid lipid ratio and establish their compatibility, different ratios of $6: 4,7: 3,8: 2$, and $9: 1$ were undertaken and evaluated for phase separation. The binary mixture was retained at an elevated temperature compared to the melting point ( $5^{\circ} \mathrm{C}$ above the melting point) of each solid lipid for the study. The mixture demonstrating no evidence of phase separation for 24 hours was selected [12].

3.1.4. Thermal Inspection of a Binary Mixture by DSC. The lipids of either category with inflated solubilization of RBO were taken. The different ratios of binary mixture with a surplus amount of liquid lipid were prepared. The ratio of soli$\mathrm{d}$ : liquid lipid in $6: 4,7: 3,8: 2,9: 1$, and $1: 0$ was prepared. The binary mixture was held onto a water bath at an elevated temperature compared to the melting point of solid lipids, i.e., $5^{\circ} \mathrm{C}$ above the melting point with continuous agitation. The final sample holds on to room temperature for 24 hours. Afterward, all binary mixture samples were analyzed using DSC (Perkin Elmer Pyres, USA) thermogram. The change in any thermal incident of solid lipid alone was considered as a control. The instrument was set at a $10^{\circ} \mathrm{C}$ heating rate per minute, and the temperature range was selected from $25^{\circ} \mathrm{C}$ to $100^{\circ} \mathrm{C}$. The crystallinity index (CI) was determined for each ratio of the binary mixture sample [13].

3.1.5. Screening of Surfactant. The optimized ratio of soli$\mathrm{d}$ : liquid lipid ( $100 \mathrm{mg}$ ) was dissolved in $3 \mathrm{ml}$ of methylene chloride. The methylene chloride was separated later by moderate heating. To the above organic mixture, $10 \mathrm{ml}$ of respective surfactant solutions of $5 \% \mathrm{w} / \mathrm{v}$ concentration was added and allowed to stir for $30 \mathrm{~min} .1 \mathrm{ml}$ of the sample was taken and further diluted to 10 times, and then, \% transmittance was taken at $510 \mathrm{~nm}$ in UV spectroscopy [13].

3.2. Preparation and Optimization of NLCs. For the development of NLCs, solvent evaporation followed by the probe sonication method was employed. The drug was dissolved in the lipidic binary mixture (solid:liquid lipid mixture) to which the surfactant was added dropwise, maintained at the same temperature. This was continuously stirred for $30 \mathrm{~min}$ at $200 \mathrm{rcf}$ followed by the probe sonication method, which was used for size reduction [11]. To obtain the optimized formulation, the QbD technique was applied wherein Box-Behnken Design (BBD) was used considering the two variables as dependent and independent. For optimization techniques, Design-Expert software was used. Three factorial designs were applied with 3 levels, which lead to a total of 17 experiments. These trials were also giving a needed quadratic equation to yield the optimized formula as undermentioned.

$$
\begin{aligned}
Y= & c_{0}+c_{1} X_{1}+c_{2} X_{2}+c_{3} X_{3}+c_{12} X_{1} X_{2}+c_{13} X_{1} X_{3}+c_{23} X_{2} X_{3} \\
& +c_{11} X_{1}^{2}+c_{22} X_{2}^{2}+c_{33} X_{3}^{2} .
\end{aligned}
$$

In the above equation, $Y$ represents the computed response association of factor level; $c_{0}$ is the constant; $c_{1}, c_{2}$, and $c_{3}$ are linear coefficients; $c_{12}, c_{13}$, and $c_{23}$ are interaction coefficients in the midst of 3 factors, whereas $c_{11}, c_{22}$, and $c_{33}$ represent observed experimental results of quadratic coefficients; and independent variable values are represented by $A_{1}, A_{2}$, and $A_{3}$ [14]. The independent variable for the said process for BBD was a binary mixture (1-3\%), surfactant \% (1$3 \%$ ), and sonication time (1-5 min). The dependent variables include the particle size as well as PDI and entrapment efficiency.

\subsection{Characterization Parameters of Optimized RBO-NLCs}

3.3.1. Particle Size and Polydispersity Index (PDI). Zetasizer Nano ZS (Malvern, UK) instrument used to determine the particle size distribution and PDI, which is based on the dynamic light scattering principle. Zetasizer calculates the variation in the intensity of light transfer attributable to the Brownian motion of the lipid nanoparticulate carrier system [15].

3.3.2. Surface Charge Determination. The surface charge of optimizing RBO-NLCs was measured in terms of zeta potential by the Zetasizer (Malvern, UK) instrument. The instrument was calibrated by the conductivity to $50 \mathrm{mS} / \mathrm{cm}$ with $0.9 \% \mathrm{w} / \mathrm{v}$ solution of sodium chloride against the purified water. The zeta potential was measured after dilution of the sample with distilled water (approx. $50 \mu \mathrm{l}$ of sample and diluted to make up to $10 \mathrm{ml}$ volume with distilled water). The diluted sample was used to determine the zeta potential at $25 \pm 2{ }^{\circ} \mathrm{C}$ [16].

3.3.3. Drug Entrapment (EE) and Drug Loading (DL). The prepared RBO-NLCs were centrifuged (Sigma, Germany) at a speed of $10,000 \mathrm{rpm}(5590 \times \mathrm{g})$ for half an hour. The supernatant was separated, and the pellets present at the bottom were collected. The pellet was further diluted with $10 \mathrm{ml}$ methanol. After dilution, mixed well and then analyzed by UV-spectroscopy method [17]. Therefore, the amount of encapsulated drug is analyzed by the amount of drug present in pellets. The following formulas were used to determine each of the parameters:

$$
\begin{aligned}
& (\%) \mathrm{EE}=\frac{W_{\text {total drug }}-W_{\text {free drug }}}{W_{\text {total drug }}} \times 100 \%, \\
& (\%) \mathrm{DL}=\frac{W_{\text {total drug }}-W_{\text {free drug }}}{W_{\text {total lipid }}} \times 100 \%,
\end{aligned}
$$

where $W_{\text {total drug }}$ was the total amount of drug, $W_{\text {free drug }}$ was the amount of unencapsulated drug, and $W_{\text {total lipid }}$ was the weight of the lipids used for NLCs preparation.

3.4. Freeze-Drying of NLCs Formulation. Mannitol was used as a cryoprotectant and added to the optimized RBO-NLCs. The obtained dispersion solution after adding mannitol was kept at a frozen temperature $\left(-20^{\circ} \mathrm{C}\right)$ overnight. Then, a sample of frozen dispersion was lyophilized at $-20^{\circ} \mathrm{C}$ for $12 \mathrm{~h}$ in a freeze dryer (Make Heto Drywinner, Denmark). The freeflowing powder was obtained after freeze-drying used for different characterization parameters during studies [18]. 
3.5. Structural Analysis by TEM. TEM (Fei Company, Netherlands) was used for the ascertainment of shape and size of optimized NLCs formulation. Before analyzing, the sample of RBO-NLCs was prepared after diluting it 10 times with distilled water onto a copper grid mesh of 400 (coated with carbon film). Then, $1 \%$ of phosphotungstic acid was added to the copper grid as negative staining. The sample above the copper grid was dried in the air. Then, the dried sample was examined in TEM. The setup of elevated magnification coupled with diffraction modes inside the instrument was used to examine the shape and size of the optimized NLCs using brightfield imaging [19].

3.6. Powder X-Ray Diffraction (PXRD) Pattern Study. For PXRD analysis, a Rigaku Ultima IV (Tokyo, Japan) instrument was used with a degree divergence slit, degree scatter slit, and receiving slit, set at $40 \mathrm{kV}$ to determine the crystallinity form of pure drug and RBO-NLCs to confirm whether the drug was entrapped into the NLCs formulation or not. Scans were performed from 10 to $80^{\circ} 2$ thetas at $8^{\circ} / \mathrm{min}$ at $25^{\circ}$ room temperature with $65-75 \%$ humidity.

3.7. Structural Analysis by FT-IR Spectroscopy. The FT-IR (Bruker, Tensor 37, Germany) spectra of drug alone and NLCs formulation were recorded. The powder sample of each sample was mixed with Potassium Bromide (KBr) in a ratio of $1: 100$. The final sample was pressed into pellets using a hydraulic pressure machine.

\subsection{Drug Release Study}

3.8.1. Activation of Dialysis Membrane. Activation of the dialysis membrane is required before in vitro study to remove glycerine and sulfur compounds and to open pores. The continuous washing for $3-4 \mathrm{~h}$ in running water removed the glycerine. The solution of $0.3 \% \mathrm{w} / \mathrm{v}$ sodium sulfide was used to wash at $80^{\circ} \mathrm{C}$ for $1 \mathrm{~min}$ to remove the sulfur compounds. The third step was to wash with hot water $\left(60^{\circ} \mathrm{C}\right)$ for $2 \mathrm{~min}$ to remove the extra sodium sulfide solution and sulfur compound. The dialysis bag was further treated with sulfuric acid $(0.2 \% v / v)$ for acidification and finally washed with hot water to remove extra sulfuric acid [20].

3.8.2. In Vitro Drug Release Study. The drug release from RBO-NLCs was performed in various buffers like $0.1 \mathrm{~N}$ $\mathrm{HCl}$ buffer $\mathrm{pH} 1.2$, acetate buffer $(\mathrm{AB}) \mathrm{pH} 4.5$, and phosphate buffer saline (PBS) pH 6.8 by dialysis technique. The $3 \mathrm{ml}$ of RBO-SUS and RBO-NLCs freshly prepared formulation was taken. Then, each sample of $3 \mathrm{ml}$ was placed in activated dialysis bags (molecular weight cut-off $12000 \mathrm{~g} / \mathrm{mole}$ ), and both ends were tied to form a sac-like structure. The dialysis bag is suspended in dissolution media of $100 \mathrm{ml}$ in the above-mentioned various buffers at $37 \pm 0.5^{\circ} \mathrm{C}$. The magnetic stirring rate was maintained at $100 \mathrm{rpm}$. The aliquot sample of $2 \mathrm{ml}$ was withdrawn at regular intervals of time from dissolution media ( $5 \mathrm{~min}, 1,2,4,6,8,12,24,32$, 48 , and $72 \mathrm{~h}$ ). The withdrawn samples were diluted with respective dissolution media. The diluted sample was used to measure the quantity of drug-using UV-spectroscopy. The drug release was calculated as per the formula given.
TABLE 1: Release kinetic equation for the analysis mechanism of drug release.

\begin{tabular}{lc}
\hline Kinetic model & Equation \\
\hline Hixson-Crowell & $3 \sqrt{ } Q_{o}-3 \sqrt{ } Q_{t}=K t$ \\
1st order & $\ln Q_{t}=\ln Q_{o}+K t$ \\
Higuchi & $Q_{o}-Q_{t}=K t^{\frac{1}{2}}$ \\
Korsmeyer-Peppas & $\log \left(Q_{o}-Q_{t}\right)=\log K+n \log t$ \\
\hline
\end{tabular}

$Q_{o}$ is the amount of drug present initially, $Q_{t}$ is the drug left over after time $t$ , and $K$ is released constantly.

The dissolution studies were performed in triplicate. The dissolution profile of each formulation was compared in terms of $\%$ dissolution efficiency (\%DE) and similarity factor (f2) [21].

$\%$ Drug release $=\frac{\text { Conc. }(\mu \mathrm{g} / \mathrm{ml}) \times \text { Dilution factor } \times \text { Volume of release medium }(\mathrm{ml})}{\text { Initial dose }(\mu \mathrm{g})} \times 100$.

3.8.3. Drug Release Kinetic Models. The release profile of the drug encapsulated into NLCs over time was used to fit into various release kinetic models like the first order, Higuchi model, Hixson-Crowell, and Korsmeyer-Peppas, as mentioned in Table 1 with their respective equations. Each equation is used to calculate the correlation coefficient $\left(R^{2}\right)$. The $R^{2}$ value close to 1 was selected as the best-fit kinetic model for drug release. The $n$ value present in the equation indicates their mechanism of drug release, which varies from 0.43 to 0.85 . If $n$ is 0.43 or less, it follows the Fickian diffusion. If $n$ value $0.43<n>0.85$, then it follows the nonFickian mechanism. If the $n$ value is 0.85 , then it follows the zero-order mechanism of drug release [22].

3.9. In Vitro Lipolysis. This study was performed to establish the solubilization potential of NLCs when given orally and the result interpreted for in vivo fate. The composition of different media used for in vitro lipolysis like digestive buffer $(17.75 \mathrm{ml})$ made of $50 \mathrm{mM}$ tris-maleate, $5 \mathrm{mM}$ calcium chloride, $150 \mathrm{mM}$ sodium chloride, and $39.75 \mathrm{mM}$ sodium hydroxide. The $\mathrm{pH}$ of the digestive buffer was adjusted to 6.8 by $1 \mathrm{M}$ sodium hydroxide. To the above buffer, $5 \mathrm{mM}$ taurocholic acid and $1.25 \mathrm{mM}$ L-alpha phosphatidylcholine were added. The resulting solution mimics the fasted state of GIT. The buffer was stirred and maintained at a temperature of $37^{\circ} \mathrm{C}$, and the $\mathrm{pH} 6.8$ was adjusted by $1 \mathrm{M}$ sodium hydroxide. The NLCs formulation of $5 \mathrm{mg}$ per $\mathrm{ml}$ drug concentration was taken and mixed to the digestive buffer and stirred for $15 \mathrm{~min}$. Another media of pancreatin extract $(1.75 \mathrm{ml})$ was added to the above digestive buffer containing the NLCs formulation made of porcine pancreatin of $200 \mathrm{mg}$ per $\mathrm{ml}$ concentration in the digestive buffer. After adding the pancreatin extract, the media were well stirred for $15 \mathrm{~min}$. The enzymatic digestion of RBO-NLCs was initiated, and accordingly, $\mathrm{pH}$ was adjusted by $0.15 \mathrm{M}$ sodium hydroxide to $\mathrm{pH}$ 6.8. The whole setup was recorded for $30 \mathrm{~min}$. The volume required to maintain the $\mathrm{pH} 6.8$ which corresponded to the release of 2 free fatty acids was calculated. The final 
TABLE 2: List of solid and liquid lipids and their solubility parameters to dissolve RBO.

\begin{tabular}{lcccr}
\hline \multirow{2}{*}{ Liquid lipids } & \multicolumn{2}{c}{ Solubility $\left(\mathrm{mg} \mathrm{mL}^{-1}\right)$} & \multirow{2}{*}{ Solid lipids } & \multicolumn{2}{c}{ Solubility $\left(\mathrm{mgg}^{-1}\right)$} \\
Mean & SD & Campritol 888 ATO & 14.00 \\
\hline Labrafil M 1944 CS & 4.6856 & 0.0605 & Precirol ATO 5 & 13.87 \\
Labrafil M 2125 CS & 10.3348 & 0.3013 & Stearic acid & 95.10 \\
Lauroglycol FCC & 2.1232 & 0.0419 & Gelot 64 & 14.70 \\
Capmul MCM- C8 & 8.1974 & 0.1463 & Capmul MCM C10 & 77.33 \\
Capmul MCM- L8 & 2.0013 & 0.1012 & Gelucire 44/14 & 2.384 \\
Capmul PG-12 & 3.3194 & 0.1596 & Gelucire 44/01 & 2.90 \\
Caprol PGE-860 & 3.3646 & 0.0818 & & 2.281 \\
Maisine 351 & 8.9596 & 0.1655 & & 2.402 \\
Peceol & 3.6687 & 0.0766 & & 2.381 \\
Plurol oleique & 11.0081 & 0.2399 & & \\
Plurol oleique CC 497 & 3.3744 & 0.1525 & & \\
Sesame oil & 4.1888 & 0.1101 & & \\
\hline
\end{tabular}

mixture was centrifuge at $5000 \mathrm{rpm}$ for $15 \mathrm{~min}$ after the end of the experiment, with the supernatant comprising the aqueous layer containing bile salts, monoglycerides, and fatty acids. The sediment comprises lipid-containing di- and triglycerides and undissolved fatty acid. The drug content was measured in each separated layer after centrifugation [23].

3.10. In Vitro Haemolysis. Haemolysis studies were performed to see the compatibility of RBCs with NLCs formulation compared to their pure drug suspension. Freshly human blood was collected in an EDTA tube and centrifuge for $5000 \mathrm{rpm}$ for $10 \mathrm{~min}$ and supernatant was decanted. The remaining solid content was washed 3 times with phosphate buffer ( $\mathrm{pH}$ 7.4). After washing with phosphate buffer, centrifuge at $5000 \mathrm{rpm}$ for $10 \mathrm{~min}$ and the solid content was taken after decantation. An appropriate amount of NLCs formulation of $20 \mu \mathrm{L}$ and diluted RBCs with phosphate buffer of $180 \mu \mathrm{L}$ were taken in 96 well plates. Similarly, $20 \mu \mathrm{L}$ of NLCs placebo, pure drug suspension, 1\% triton X100 (negative control) and phosphate buffer (positive control) were taken at respective 96 well plates. The optical density was measured by the Elisa reader at $570 \mathrm{~nm}$ after one hour from the sample added to diluted RBCs (RBCs in phosphate buffer). Furthermore, percentage haemolysis was calculated by the given formula [24]:

$$
\text { Haemolytic activity }(\%)=\left\{\frac{\mathrm{As}-\mathrm{Ab}}{\mathrm{Ac}}\right\} 100
$$

where As, Ab, and Ac are the optical density of the sample, placebo, and positive control, respectively.

3.11. In Vitro Gut Permeation Study. The small intestine of Wistar rat (Protocol No. 1587. from IAEC, Jamia Hamdard) was used for in vitro gut permeation study, which was fasted overnight. All animal experiments comply with the ARRIVE guidelines. The $\mathrm{CO}_{2}$ inhalation is used to sacrifice the rat. The small intestine was isolated into $4-5 \mathrm{~cm}$ length. The isolated intestine was washed with Tyrode buffer $\mathrm{pH} 7.4$ to clean the content of the intestine. The intestinal part of

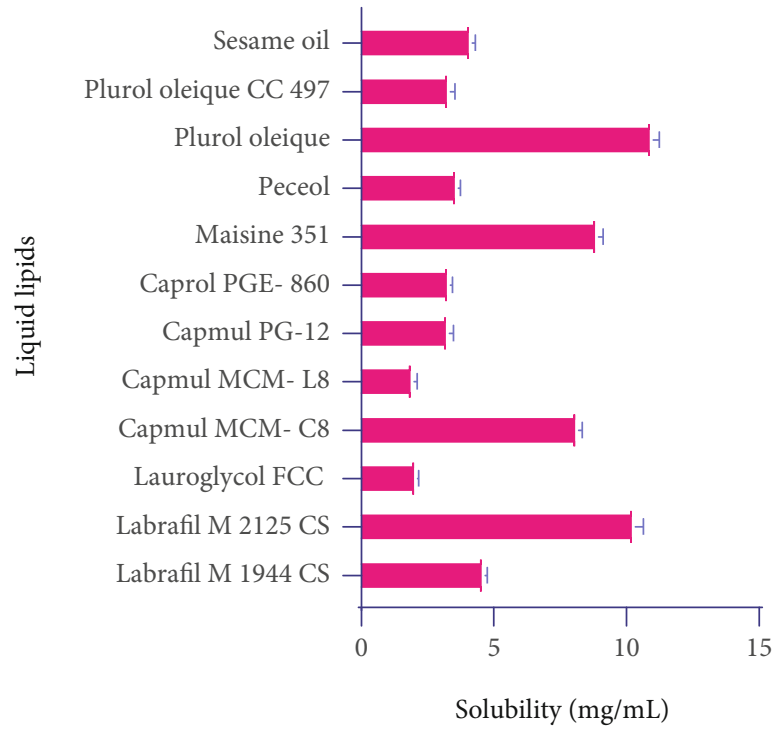

Figure 2: Solubility of RBO in liquid lipids.

one end is tied with thread, and $3 \mathrm{ml}$ of RBO-NLCs is filled using a syringe from the second end and tied. The sac formed after tying from both ends is immersed into a glass beaker maintained at $37^{\circ} \mathrm{C}$, which contains oxygenated Tyrode buffer pH7.4 (100 ml). The aliquot of $2 \mathrm{ml}$ was withdrawn at regular intervals of time like $5 \mathrm{~min}, 10 \mathrm{~min}$, $15 \mathrm{~min}, 30 \mathrm{~min}, 1 \mathrm{~h}, 1.5 \mathrm{~h}$, and $2 \mathrm{~h}$ and replaced with similar preheated dissolution media.

Similarly, the experiment was done for RBO-SUS. The aliquot taken from each sample at regular intervals of time was analyzed for drug content by HPLC [25]. The flux and apparent permeability coefficient were calculated using the following formula:

$$
\begin{gathered}
\text { Flux of drug }=\frac{\text { Conc } .(\mu \mathrm{g} / \mathrm{ml}) \times \text { Dilution factor } \times \text { Volume of release medium }(\mathrm{ml})}{\text { Permeation area }\left(\mathrm{cm}^{2}\right)} \times 100, \\
\text { Apparent permeability coefficient, } P\left(\mathrm{~cm} \mathrm{~min}^{-1}\right)=\frac{J}{C_{o}},
\end{gathered}
$$




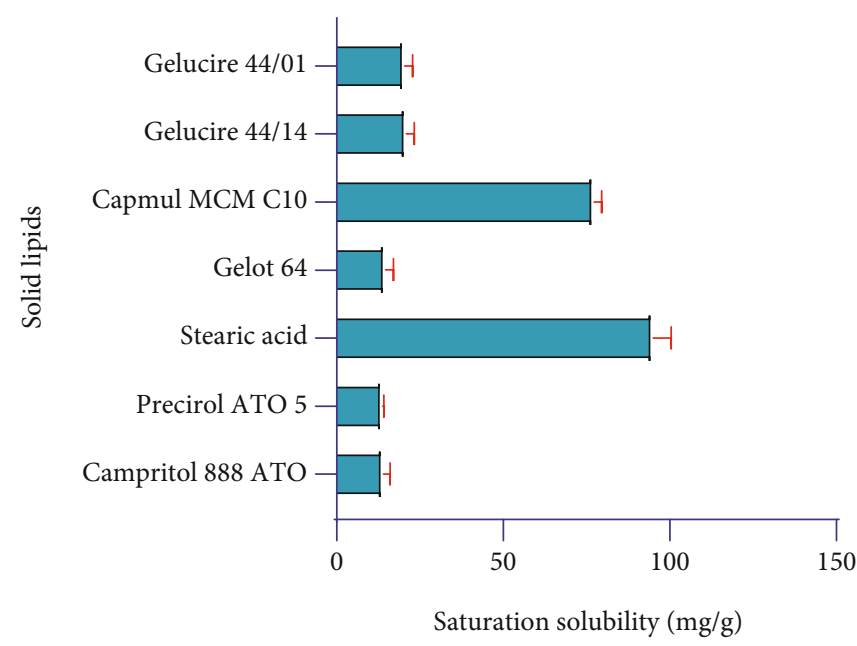

FIgURE 3: Solubility of RBO in solid lipids.

TABLE 3: Results of miscibility study between liquid lipids and solid lipids.

\begin{tabular}{lcclc}
\hline Solid lipid & Liquid lipid & Observation & Inferences \\
\hline \multirow{4}{*}{ Stearic acid } & Labrafil M 2125 CS & After 48 h, separate layers were observed. & Failed \\
& Capmul MCM-C8 & Uniform distribution and no phase separation were observed. & Good compatibility \\
& Maisine 351 & Turbidity and phase separation was found after $48 \mathrm{~h}$. & Failed \\
& Plurol oleique & & Passed \\
Labrafil M 2125 CS & No phase separation was observed. & Failed \\
\hline
\end{tabular}

where $C_{o}$ is the initial quantity of the drug present in the donor compartment.

3.12. Depth Permeation Study by Confocal Microscopy. The experiment assessed the depth of penetration of RBONLCs compared to drug suspension in small intestinal gut cells. The NLCs formulation and drug suspension were added with Rhodamine B dye $(0.03 \%)$ during the preparation of each formulation. The experiment kept for $2 \mathrm{~h}$ as mentioned in section 3.11. Then intestinal gut samples were separated and cleaned with Tyrode solution, $\mathrm{pH}$ 7.4. The cleaned intestinal gut was subjected cut off into thin sections and fixed over the slide for further analysis. The thin section was examined by confocal microscopy (LEICA TCS SPEII Leica Microsystem Ltd., Germany) with LAS AF software. Fluorescence indicator from cells of the intestine was examined at various depths of the intestinal membrane. The magnitude and depth of permeation of fluorescence in intestinal membrane cells were calculated through the $z$-axis and compared the result [26].

\subsection{Cell Line Study}

3.13.1. Cell Line and Cell Culture. The MCF-7 BC cell lines purchased from National Centre for Cell Science, Pune, India (Job No. 408/20220.21), were cultured in DMEM supplemented with composition $10 \%(v / v)$ fetal bovine serum (FBS) and $1 \%(0.01 \mathrm{~g} / \mathrm{mL})$ penicillin-streptomycin solution maintained at $37^{\circ} \mathrm{C}$ temperature in a humidified incubator of $<5 \% \mathrm{CO}_{2}$ and $95 \%$ humidity.

3.13.2. Determination of Cell Cytotoxicity by MTT Assay. The cytotoxicity of the RBO at concentrations of $12.5,25$, 50 , and $100 \mu \mathrm{M}$ was evaluated as per the previously published protocol, and percent cytotoxicity was calculated in the MCF7 cell line. Briefly, $5 \times 10^{3}$ cells per well were seeded in a 96well microtiter plate containing $10 \% \mathrm{FBS}$ and kept for incubation for $24 \mathrm{hrs}$ maintained at $37^{\circ} \mathrm{C}$ with $5 \% \mathrm{CO}_{2}$. The next day, the cells were treated with samples at mentioned concentrations for $24 \mathrm{hrs}$ and $48 \mathrm{hrs}$. The $20 \mu \mathrm{l}$ of MTT solution made in PBS pH7.4 of $5 \mathrm{mg} / \mathrm{ml}$ concentration was added to each well and remained for $4 \mathrm{hrs}$ at $37^{\circ} \mathrm{C}$ which allowed the formation of formazan crystals. The crystals were again dissolved in DMSO by adding $150 \mu \mathrm{l}$ to each well. Finally, the content of the plate was well mixed on mechanical plate mixer and then optical density (OD) was measured in an ELISA reader (Synergy HT, BioTek, USA) at $570 \mathrm{~nm}$. The colour developed in each well is directly related to the viable present in respective well. The $50 \%$ inhibition concentration $\left(\mathrm{IC}_{50}\right)$ was determined after $24 \mathrm{hrs}$ and $48 \mathrm{hrs}$, and percentage cytotoxicity was calculated as per the given formula. All experiments were done in triplicates [27].

$$
\% \text { cytotoxicity }=\frac{[\mathrm{C}]_{\text {control }}-[\mathrm{T}]_{\text {test }}}{[\mathrm{C}]_{\text {control }}} \times 100 \text {, }
$$



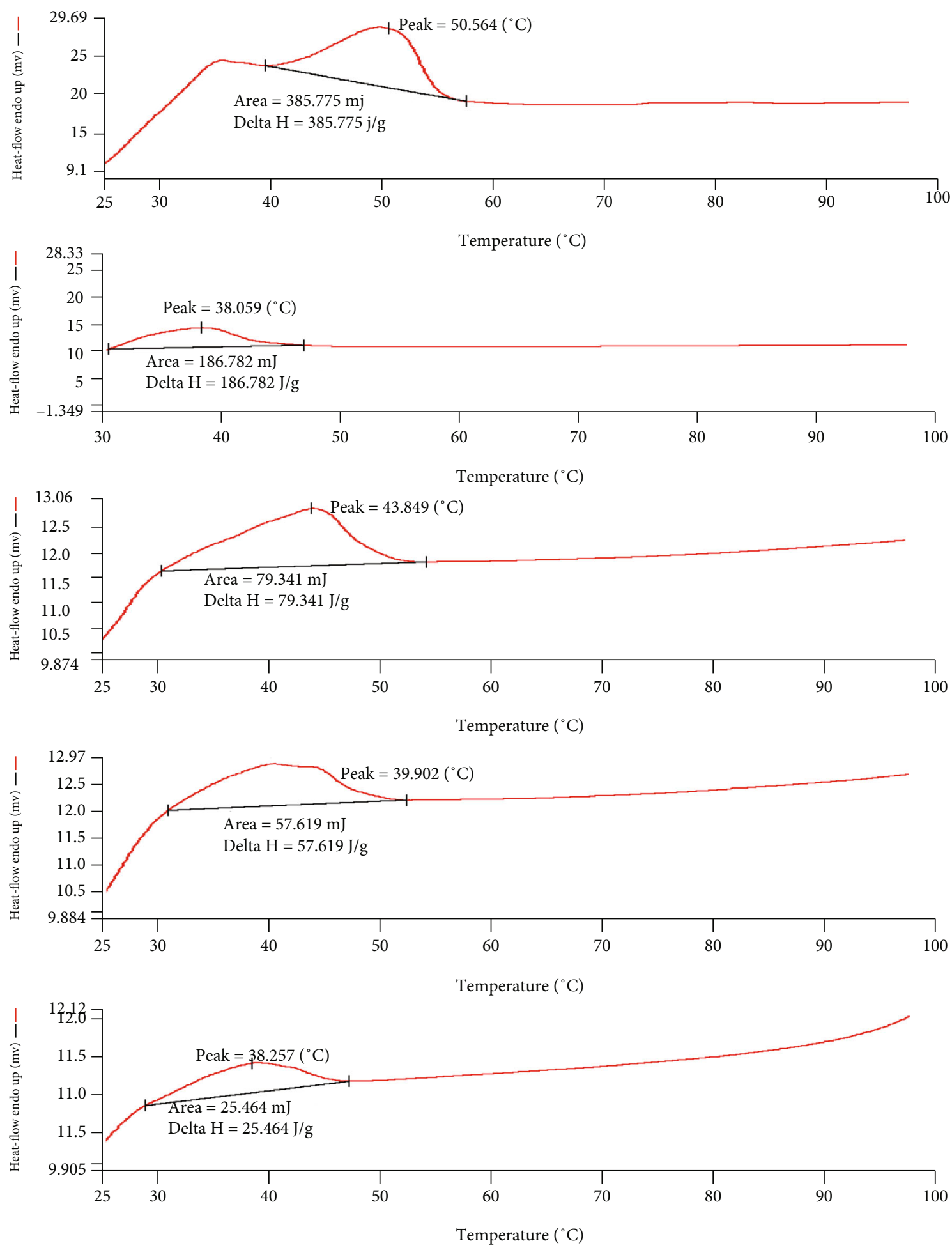

FIGURE 4: Compatibility study of liquid lipids and solid lipids.

where $[T]_{\text {test }}$ is the Absorbance of test sample and $[\mathrm{C}]_{\text {control }}$ is the absorbance of the control sample.

The concentration value of $\mathrm{RBO}$ needed to inhibit the cell growth by $50 \%$ so that IC50 values were calculated using a dose-response curve.
3.14. In Vivo Pharmacokinetic Study. All experiments on the animal were conducted as per the guidelines of IAEC, Jamia Hamdard, New Delhi, India (Protocol no. 1587). The animals were kept under normal laboratory supply, i.e., temperature of $25 \pm 2^{\circ} \mathrm{C}$ and relative humidity of $55 \pm 5 \% \mathrm{RH}$ with 
normal laboratory diet supply. For pharmacokinetic study, female Wistar rats were assigned randomly into two groups $(n=3)$. Among two groups, group A received RBO suspension (approx. $20 \mathrm{mg} / \mathrm{kg}$ of body weight) and group B received RBO-NLCs (approx. $20 \mathrm{mg} / \mathrm{kg}$ of body weight) calculated from human to animal dose conversion factor [28]. RBO being a water insoluble compound, the suspension of $\mathrm{RBO}$ was made with $0.25 \% w / v \mathrm{Na}$-CMC (suspending agent) with a constant stirring for $10 \mathrm{~min}$ so that the final concentration of suspension was $2.5 \mathrm{mg} / \mathrm{ml}$ of RBO. The similar method of suspension preparation has been reported by Singh and associates in which $\mathrm{Na}-\mathrm{CMC}$ was added to water at temperature of 40 to $50^{\circ} \mathrm{C}$ under continuous stirring of $600 \mathrm{rpm}$, and the same has been followed for the current study [26].

Blood sample were collected from venous blood drawn from the lateral tail vein in EDTA-coated tubes in predetermined interval of times $(0.5,1,2,4,8,12,24$, and $48 \mathrm{~h})$ under mild $\mathrm{CO}_{2}$ inhalation anaesthesia. The blood samples were centrifuged for $0.5 \mathrm{~h}$ at $5000 \mathrm{rpm}$ to separate plasma from blood. The plasma samples were stored at $-80^{\circ} \mathrm{C}$ until the further analysis by HPLC. The plasma samples were thawed out before analysis. For deproteinization, the plasma samples were mixed with acetonitrile (equal quantity of plasma so that $50 \%$ dilution). Then, the samples were centrifuged for $0.25 \mathrm{~h}$ at $5000 \mathrm{rpm}$, and supernatants were collected for quantification of RBO by HPLC [26].

3.15. Stability Study. The optimized RBO loaded into NLCs was stored at room temperature with set down conditions of $25 \pm 2{ }^{\circ} \mathrm{C} / 60 \pm 5 \%$ relative humidity as per Q1A(R2), $\mathrm{ICH}$ guideline. The change in any physical parameters like particle size and PDI and chemical parameters like entrapment efficiency monitored during the storage period of 6 months [29]

\section{Results and Discussions}

\subsection{Assessment of Excipients}

4.1.1. Selection of Lipids. The selection of different required ingredients like lipids for the fabrication of NLCs was performed based on the maximum solubility of the drug in each ingredient. The solubility of RBO performed in several lipids and results are elaborated in Table 2 and Figures 2 and 3.

Based on solubility data, Labrafil M2125 CS (HLB 4), Capmul MCM C8 (HLB 5-6), Maisine 3S1 (HLB 4), and Plurol oleique (HLB 6) were showing high solubility of $\mathrm{RBO}$ as it was anticipated that the structure containing carboxylic acid and succinate group favors the solubility of RBO in each liquid lipid. In addition, longer triglycerides have shown more solubility than the medium-chain due to more entrapment of drugs. Similarly, Stearic acid (HLB 17) and Capmul MCM C10 (HLB 5) have shown high solubility of RBO due to similar reasons. The solubility parameters of each lipid direct reflect the encapsulation efficiency. Thus, the high solubility associated with the drug in lipids is also attributed to the high encapsulation efficiency of the NLCs formulation [29]. Bang and coworkers investigated NLCs of anticancer drug paclitaxel, and similar methods were

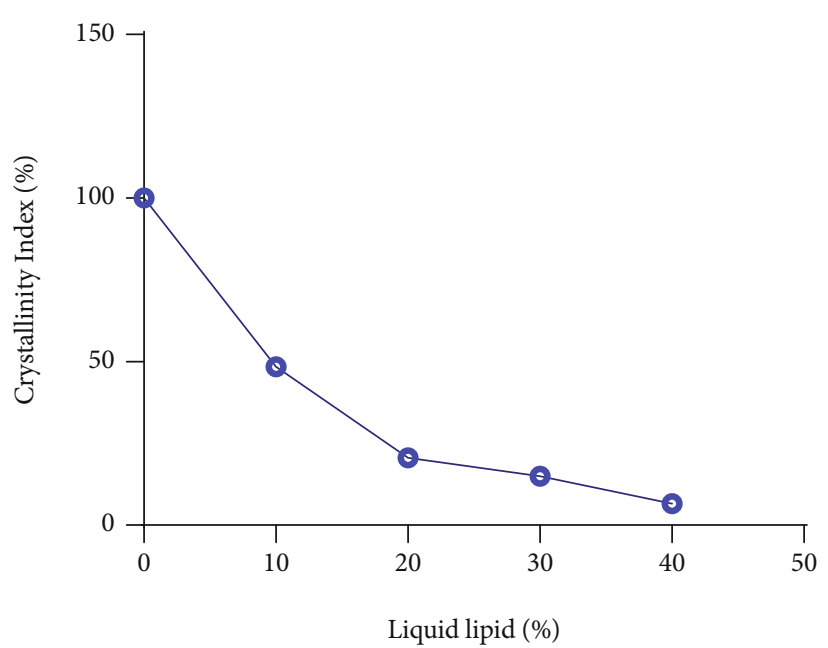

FIgURE 5: Determination of compatibility of lipids through Crystallinity Index (\%).

TABLE 4: Observational study for the selection of surfactants.

\begin{tabular}{lc}
\hline Name of surfactants & \% transmittance \\
\hline Cremophor EL & 64.23 \\
Cremophor RH 40 & 91.61 \\
Poloxamer 188 & 92.04 \\
Solutol HS 15 & 98.28 \\
Tween 20 & 45.95 \\
Tween 80 & 63.69 \\
\hline
\end{tabular}

applied for the selection of solid and liquid lipid for the NLCs preparation $[30,31]$.

4.1.2. Compatibility Study of Lipids. The results of compatibility studies are given in Table 3. Labrafil M 2125 CS, Capmul MCM- C8, Maisine 351, Plurol oleique were selected as liquid lipids, and Stearic acid and Capmul MCM C10 were selected as solid lipids for further compatibility/miscibility study among lipids. Negi and coworkers develop a protocol for solid and liquid lipid-based compatibility on miscibility/affinity and the physical compatibility of lipids. So based on these parameters, Capmul MCM C8 and Compritol were selected [31].

4.1.3. Thermal Inspection of a Binary Mixture by DSC. In a ratio of the binary mixture, as the \% of liquid lipid increases by $10 \%$, the enthalpy for the melting point decreases in respect of the solid lipid (100\%) as shown in Figure 4. However, the crystallinity index (CI) has less variability in the ratio of $8: 2$ to $6: 4$ compared to $9: 1$. Therefore, the final ratio was selected based upon CI, i.e., 6:4, as shown in Figure 5 [32]. Iqbal and associates reported similar parameters for the selection of the best ratio of solid and liquid lipids for a suitable binary mixture. As the \% of the liquid lipid (Sefsol 218) increases, the enthalpy of the binary mixture decreases with respect to the solid lipid (Geleol), and 
TABLE 5: Quality target product profile of the nanostructured lipidic carrier.

\begin{tabular}{|c|c|c|}
\hline QTPP parameters & Target to achieve & Justification \\
\hline Dosage type & Nanoformulation & Escalate the drug permeation/bioavailability \\
\hline Route of administration & Oral & Easy to use/lymphatic uptake/stability/minimize side effects \\
\hline \multirow[t]{2}{*}{ Physical state } & Lyophilized powder & Easy administration/physical aspect \\
\hline & Entrapment efficiency & Drug loading assurance \\
\hline \multirow[t]{3}{*}{ Physiochemical characterization } & Particle size & Influence the permeation and absorption \\
\hline & Zeta potential & Product stability assurance \\
\hline & Absorption & \\
\hline \multirow[t]{2}{*}{ Pharmacokinetics } & Distribution & Required to achieve the desired efficacy \\
\hline & Metabolism and targeting & \\
\hline
\end{tabular}

TABLE 6: List of various CQAs affecting the therapeutic efficacy of RBO-NLCs.

\begin{tabular}{lcc}
\hline CQA parameters & Target to achieve & Justification \\
\hline Particle size & $100-200 \mathrm{~nm}$ & Assure the absorption, hence the increase in bioavailability \\
Entrapment efficiency & $\geq 70 \%$ & To reach the optimum therapeutic efficacy \\
PDI & $\leq 0.7$ & Uniform drug distribution, hence content uniformity \\
\hline
\end{tabular}

Cause and effect (Ishikawa or Fishbone) diagram

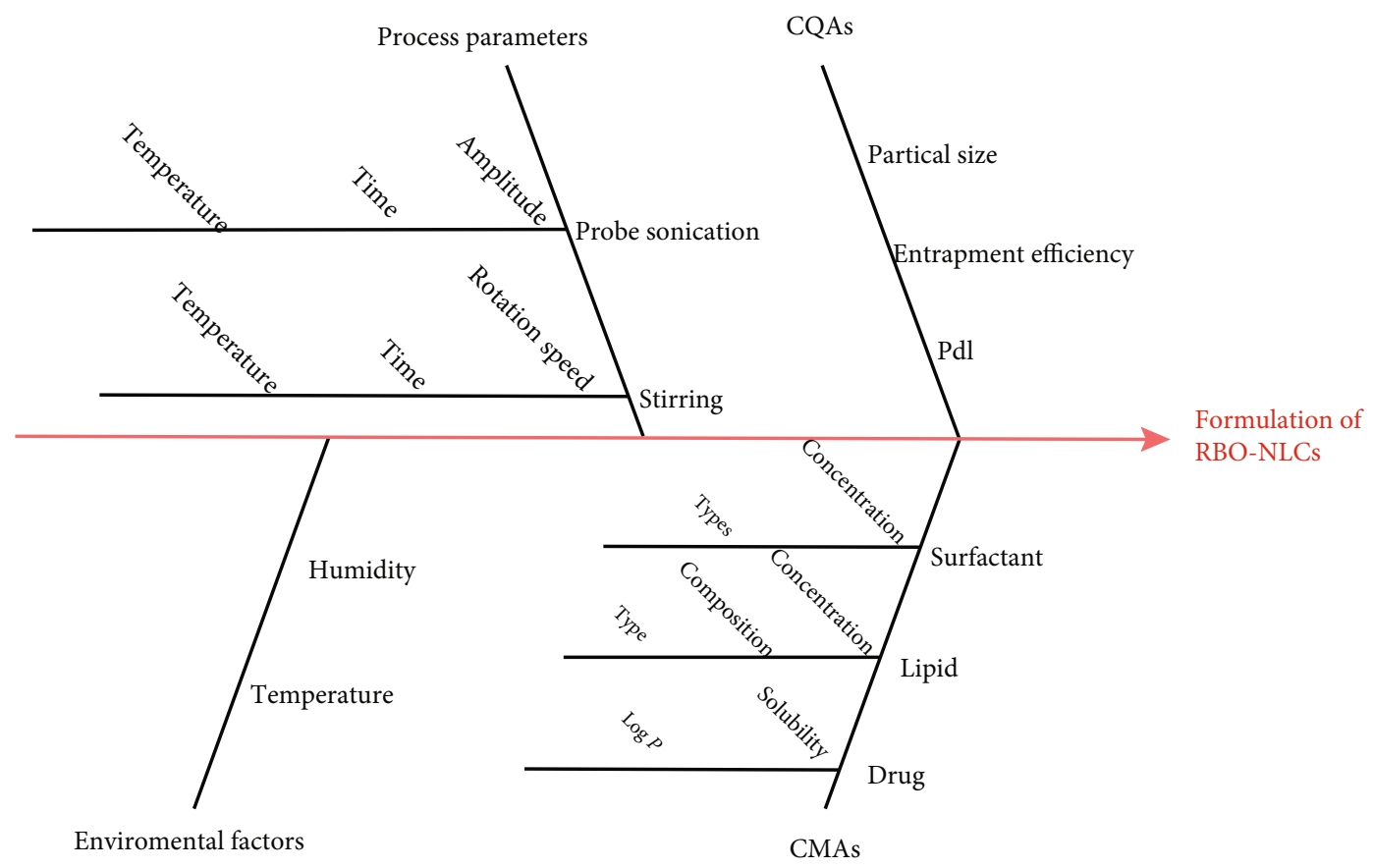

FIgURE 6: Ishikawa diagram to understand the relation of various factors.

it was accompanied by a decrease in crystallinity index from 100.0 to $30.3 \%$ [13].

4.1.4. Selection of Surfactant. The surfactants, namely, Cremophor RH 40, Cremophor EL, Tween 20, Tween 80, Poloxamer 188, and Solutol HS15, were used to evaluate for its emulsification property. The result of the screening is illus- trated in Table 4. The higher emulsification property reflects to reduce the particle size of binary mixture lipids and increase the physical stability of lipid nanoparticles, sequentially preventing the aggregation of nanoparticles which gives higher transmittance [33]. Alam and coworkers developed isradipine NLCs. They have reported in their research about the surfactant selection criteria based on $\%$ of 
Table 7: Different variables selected for Box-Behnken design.

\begin{tabular}{|c|c|c|c|c|}
\hline \multirow{2}{*}{ Independent variables } & \multirow{2}{*}{ Factors } & \multirow{2}{*}{ Unit } & \multicolumn{2}{|c|}{ Level } \\
\hline & & & Low & High \\
\hline $\mathrm{A} 1$ & Binary mixture & $\% w / v$ & 1 & 3 \\
\hline A2 & Surfactant & $\% w / v$ & 1 & 3 \\
\hline A3 & Sonication & Min & 1 & 5 \\
\hline
\end{tabular}

TABLE 8: Experimental runs and observed responses from BBD.

\begin{tabular}{|c|c|c|c|c|c|c|c|}
\hline Std & Runs & $\begin{array}{c}\text { Factor } 1 \\
\text { Binary mixture }(\% w / v \\
)\end{array}$ & $\begin{array}{c}\text { Factor } 2 \\
\text { Surfactant }(\% w / \\
v)\end{array}$ & $\begin{array}{c}\text { Factor } 3 \\
\text { Sonication time } \\
(\mathrm{min})\end{array}$ & $\begin{array}{l}\text { Response } 1 \\
\text { Particle size } \\
\quad(\mathrm{nm})\end{array}$ & $\begin{array}{c}\text { Response } \\
2 \\
\text { PDI }\end{array}$ & $\begin{array}{c}\text { Response } 3 \\
\text { Entrapment efficiency (\%w/ } \\
w)\end{array}$ \\
\hline 14 & 1 & 3 & 1 & 3 & 656 & 0.653 & 94.5 \\
\hline 13 & 2 & 2 & 2 & 3 & 211.9 & 0.562 & 89.9 \\
\hline 17 & 3 & 2 & 3 & 5 & 179.87 & 0.554 & 89.99 \\
\hline 16 & 4 & 1 & 2 & 1 & 230.7 & 0.497 & 87.1 \\
\hline 1 & 5 & 1 & 1 & 3 & 212.54 & 0.499 & 85.9 \\
\hline 9 & 6 & 1 & 2 & 5 & 152.32 & 0.618 & 86.1 \\
\hline 3 & 7 & 2 & 3 & 1 & 221.92 & 0.5 & 87.76 \\
\hline 15 & 8 & 3 & 3 & 3 & 276.53 & 0.621 & 96.12 \\
\hline 8 & 9 & 2 & 2 & 3 & 225.9 & 0.549 & 87.98 \\
\hline 11 & 10 & 2 & 2 & 3 & 234.8 & 0.541 & 89.42 \\
\hline 7 & 11 & 2 & 1 & 5 & 465.71 & 0.487 & 87.91 \\
\hline 12 & 12 & 2 & 2 & 3 & 212.7 & 0.502 & 88.4 \\
\hline 6 & 13 & 1 & 3 & 3 & 114.23 & 0.649 & 87.7 \\
\hline 4 & 14 & 3 & 2 & 5 & 543.73 & 0.597 & 95.4 \\
\hline 5 & 15 & 3 & 2 & 1 & 419.34 & 0.682 & 96.71 \\
\hline 2 & 16 & 2 & 2 & 3 & 211.9 & 0.562 & 89.9 \\
\hline 10 & 17 & 2 & 1 & 1 & 396.4 & 0.512 & 90.14 \\
\hline
\end{tabular}

TABLE 9: Interpretation of regression value of dependent variable responses B1, B2, and B3.

\begin{tabular}{|c|c|c|c|c|c|c|}
\hline \multirow{2}{*}{ Dependent variables } & \multirow{2}{*}{$R^{2}$} & \multicolumn{2}{|c|}{$R^{2}$} & \multirow{2}{*}{ Adequate precision } & \multirow{2}{*}{$\mathrm{SD}$} & \multirow{2}{*}{$\% \mathrm{CV}$} \\
\hline & & Adjusted value & Predicted value & & & \\
\hline Particle size (B1) & 0.9983 & 0.9962 & 0.9908 & 74.6866 & 9.27 & 3.17 \\
\hline PDI (B2) & 0.9500 & 0.8856 & 0.7640 & 12.2250 & 0.0211 & 3.75 \\
\hline EE (B3) & 0.9692 & 0.9297 & 0.7403 & 14.2001 & 0.9234 & 1.03 \\
\hline
\end{tabular}

transmittance, which was examined by UV spectroscopy method at $510 \mathrm{~nm}$ [12].

4.2. Preparation and Optimization of NLCs. With regard to approaches to Pharmaceutical Development, the product should be designed to meet needs and the intended product performance. A researcher might select either an experimental approach, an organized approach, or both for product development. A more organized approach for product development also refers to the quality by design approach, which includes adding prior knowledge and their results using the design of experiments (DOE). Such an organized approach helps to get the final desired product with the desired quality. It also helps to understand all the regulations. With respect to the quality of the product, which is generally related to safety and efficacy, setup parameters were justified, which refers to the quality target product profile (QTPP) which gives the outlook summary of drug product characterization, which can be predetermined. The dependent variables like particle size, entrapment efficiency, and PDI are considered critical quality attributes (CQAs). For effective delivery at the targeted site, the particle size should be small; more entrapment of the drug leads to therapeutic efficacy even at a low dose and low PDI $(\leq 0.7)$ [34] which is 


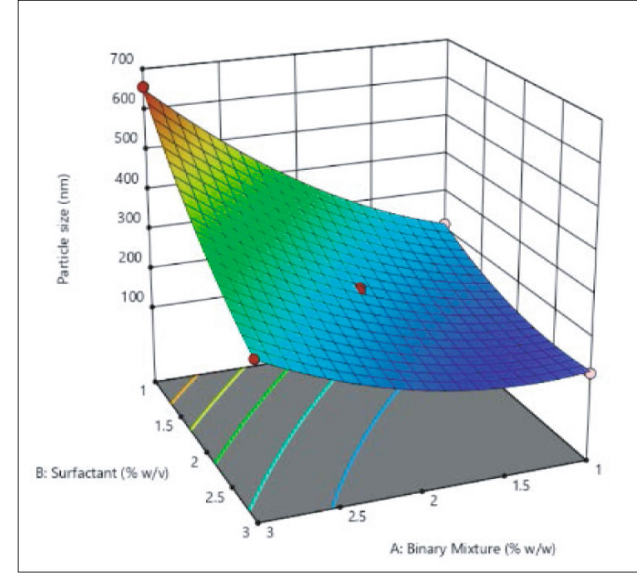

(a)

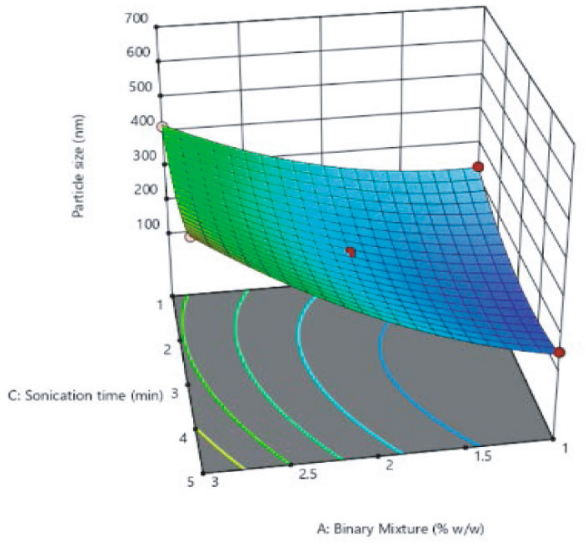

(b)

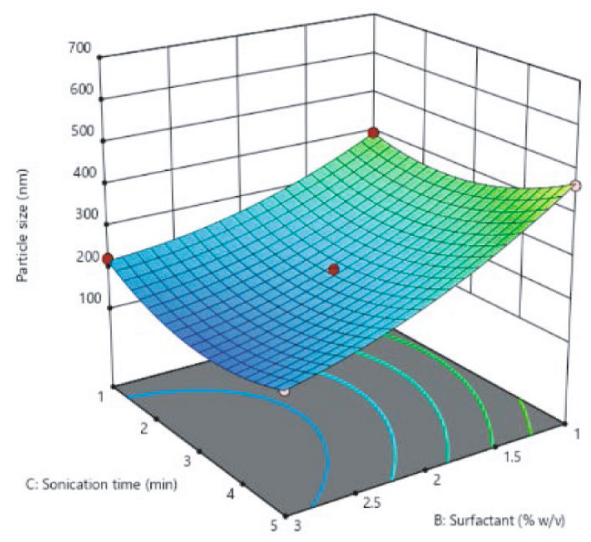

(c)

FiguRE 7: Effect of independent variables: (a) binary mixture and surfactant; (b) binary mixture and sonication; (c) surfactant and sonication on particle size.

considered as a uniformly distributed formulation. These parameters of QTPP and CQAs are summarized in Tables 5 and 6, respectively $[35,36]$.

4.2.1. Risk Assessment. The profound parameters for further experiment for optimization of formulation using BoxBehnken design from preliminary assessed data were binary mixture concentration (1-3\%), surfactant concentration (1$3 \%$ ), and sonication time (1-5 min). The $100 \%$ equipped response surface fraction is present in the design space to signify its accuracy all over the region. Figure 6 demonstrated the relation among all factors for NLCs preparation prepared by software Minitab 19, Philadelphia, USA. Similarly, Kovacs and associates have reported for the salicylic acid-loaded NLCs in which they have investigated the risk assessment, which referred to both qualitative and quantitative estimates of the risk. An Ishikawa diagram was drawn to find out the key material and process attributes which affects the NLCs formulation preparation [37].

4.2.2. Box-Behnken Design (BBD). The Box-Behnken design (BBD) response surface method tested the points which are within a predetermined range unlike the central composite design (CCRD) to get the total possible runs based on the optimized NLCs formulation data, which was prepared by probe sonication technique; 17 runs were obtained using the mentioned variables in Table 7 . Table 8 gives the result derived from the experimental runs. The optimized formula obtained was a binary mixture, surfactant, and sonication time which were found as $1 \% w / v, 3 \% w / v$, and $3 \mathrm{~min}$, respectively. The results of the dependent variable for the optimized formula were particle size $(114.23 \pm 2.75 \mathrm{~nm})$, entrapment efficiency $(87.7 \pm 1.79 \% w / w)$, and PDI $(0.649 \pm 0.043)$.

The quadratic equations obtained for different dependent variables and $p$ value were found significant, and lack of fit has been found to be insignificant, as mentioned below in Table 9.

Particle Size $(B 1)=219.44, X 148.23, Y-117.26, Z$ 9.16, $X Y-70.29, X Z$ 50.69, YZ -27.84, $X^{2} 57.97, Y^{2} 37.42, Z^{2} 59.12$

PDI $(B 2)=0.5432, X 0.0363, Y 0.0216, Z 0.0081, X Y$ -0.0455, XZ -0.0515, YZ 0.0198, $X^{2} 0.0738, Y^{2}-0.0115, Z^{2}$ $-0.0185$

Entrapment efficiency $(B 3)=89.12, X$ 4.49, $Y$ 0.3900, $Z$ -0.2888, $X Y-0.0450, X Z-0.0775, Y Z 1.11, X^{2} 2.16, Y^{2}$ $-0.2213, Z^{2} 0.0513$ 


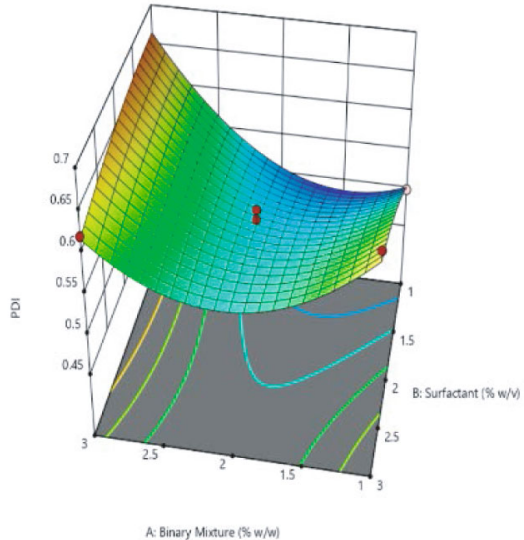

(a)

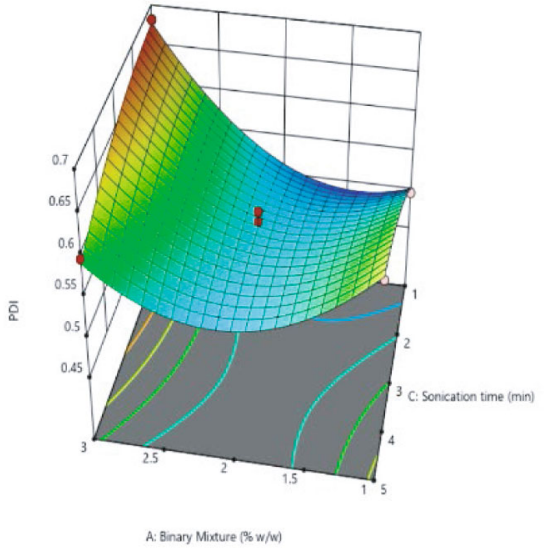

(b)

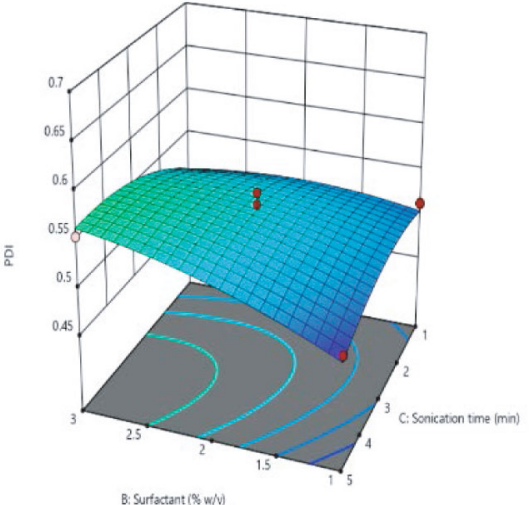

(c)

FIGURE 8: Effect of independent variables: (d) binary mixture and surfactant; (e) binary mixture and sonication; (f) surfactant and sonication on PDI.

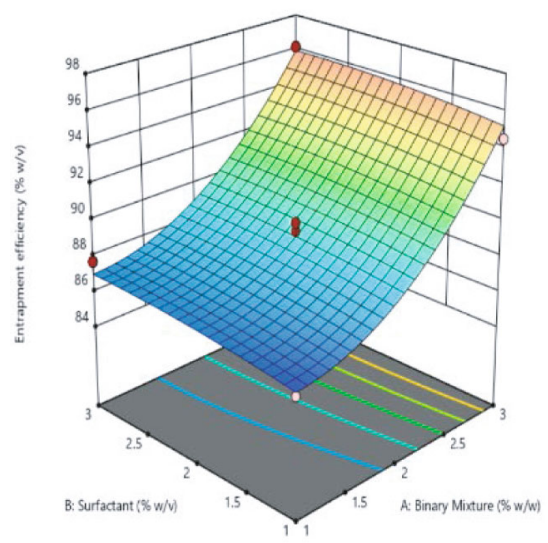

(a)

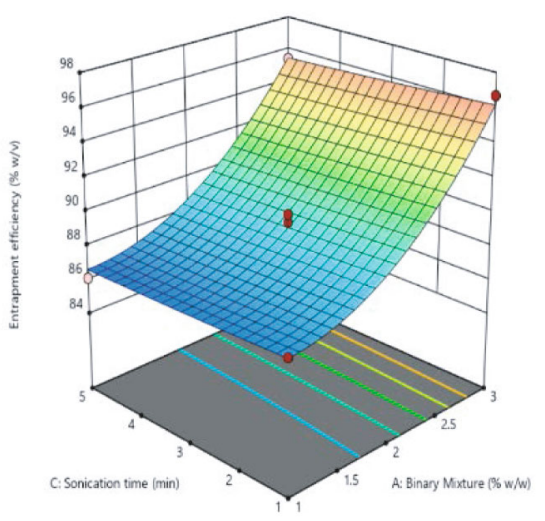

(b)

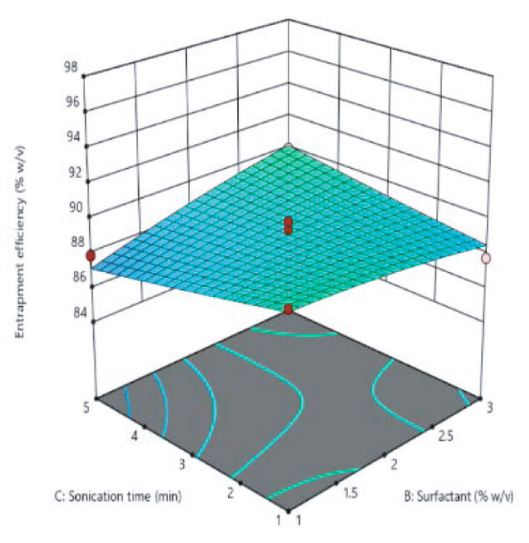

(c)

FIGURE 9: Effect of independent variables: (g) binary mixture and surfactant; (h) binary mixture and sonication; (i) surfactant and sonication on entrapment efficiency.

In all the equations, $A$ represents the binary mixture $\%, B$ is the surfactant $\%$, and $C$ depicts the sonication time (in $\min$ ). The effect between each category of variables was depicted using 3-dimensional graphs in response surface analysis, as shown in (Figures 7, 8, and 9).

\subsubsection{Impact of Independent Variables on Responses}

(1) Impact of the Binary Mixture on Dependent Variables. As the binary mixture concentration increases, the particle size also increases because of agglomeration of particles due to lack of emulsifying agent (surfactant) as more emulsifying agent is required if lipid concentration increases aqueous phase, and lack of it is attributed to an increase in particle size.

Entrapment efficiency was directly affected binary mixture quantity. This indicated that an increase in the lipid concentration leads to an increase in the amount of solid lipid, which can be, accommodated more drugs inside the
NLCs formulation in the solid lipid [38]. Similarly, Subedi and associates investigated the Doxorubicin loaded into the lipidic carrier, which stated the factor affecting the quantity of lipid on particle size as well as encapsulation efficiency. So, the increment of the lipid content increases the encapsulation efficiency and particle size [39].

(2) Impact of Surfactant on Dependent Variables. The lipid nanoparticle particle size decreases as the concentration of surfactant increases above the critical micelle concentration due to the decrease in interfacial tension. This critical concentration of surfactant also forms a network on the surface of lipid nanoparticles which prevent its agglomeration of the small particle from forming a bigger particle size.

However, the increase in the concentration of surfactant on entrapment efficiency was found very minimal [40]. The results are in agreement with the research reported by Shtay and associates. They have studied the factorial design, which 
TABLE 10: Point prediction method to attain predicted and actual response (maximum entrapment efficiency, optimum PDI, and minimum particle size) [12].

\begin{tabular}{|c|c|c|c|c|c|c|}
\hline & Lipid $(\% w / v)$ & Surfactant $(\% w / v)$ & Sonication time (min) & Particle size (nm) & Entrapment efficiency (\%) & PDI \\
\hline Predicted & \multirow{2}{*}{1.0} & \multirow{2}{*}{3.0} & \multirow{2}{*}{3.0} & 219.44 & 89.12 & 0.543 \\
\hline Actual & & & & 117.00 & 87.70 & 0.600 \\
\hline
\end{tabular}

applied to variables that affecting their preparation for food application using lipid nanocarrier. They have reported the increase in concentration decrease the particle size explained by a decrease in interfacial tension [40].

(3) Impact of Sonication Time on Dependent Variables. The mechanism of probe sonication is based on the principle that high energy waves ultrasonically emitted cause the breakage of lipid nanoparticles into small nanoparticles. Thus, upon an increase in sonication time, the lipid nanoparticle size decreases and forms monodisperse globules because of the shear force generated by cavitation.

However, the impact of sonication on entrapment efficiency was observed to be very minimal with aggravation in the sonication time [41]. Ahad and coworkers formulate the lipidic nanocarrier using an experimental design technique for valsartan. They reported an inverse relationship between the sonication time and vesicle size [42].

4.2.4. Validation of Design of Experimental. The goal of the current study is to achieve minimum particle size with maximum entrapment efficiency. Design-Expert software, version 12.0.3.0 (Minneapolis, USA), was applied for the predicted value of response given in Table 10. Based on independent variable values, RBO-NLCs prepared and compared their data with a predicted response. The value obtained from the experiment was almost similar, which confirms the validity of the optimized process for preparing NLCs except for the particle size, which might be due to the wide variety of particle data obtained from a number of runs which give a broad mean value.

\subsection{Characterization of Optimized RBO-NLCs}

4.3.1. Particle Size, PDI, and Surface Charge. The nanoparticle size (Z-Average) and PDI were obtained at $114.23 \pm$ $2.75 \mathrm{~nm}$ and $0.649 \pm 0.043$, respectively. The zeta potential of RBO-NLCs was obtained at $2.61 \pm 0.54 \mathrm{mV}$. Smaller particle size suggests good miscibility and more systemic circular time. The low PDI value states the uniformity of nanoparticle distribution. Zeta potential indicates the stability of the nanoformulation. Varshosaz and coworkers investigated the NLCs preparation in which the particle size found was smaller (103 to $127 \mathrm{~nm}$ ) because their good miscibility of the excipient and zeta potential were positive values suggesting longer residence time and less uptake by the reticuloendothelial system in the blood circulation system [43].

4.3.2. Drug Entrapment (EE) and Drug Loading (DL). The \% drug entrapment efficiency of NLCs was calculated to be $87.7 \pm 1.79 \% w / w$. The lipophilic drug has the intrinsic prop-

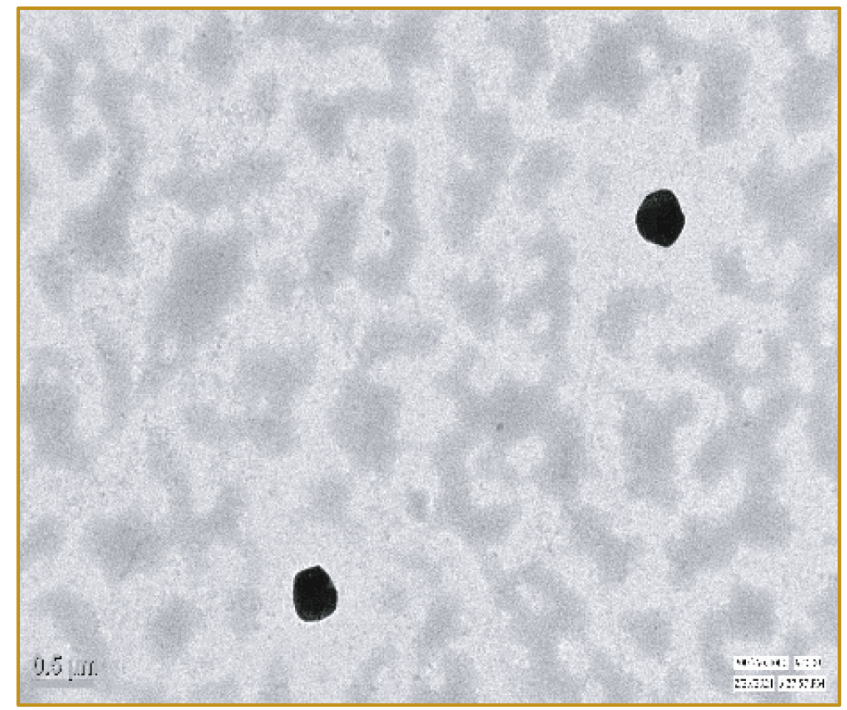

FIGURE 10: TEM image of optimized RBO-NLCs.

erty to get more solubilization in lipids, unlike the hydrophilic drug. So, this lipophilic property entraps them more inside the lipids.

The $\% \mathrm{DL}$ was found to be $8.77 \pm 0.18 \% w / w$. Elmowafy and coworkers investigated drug-loaded NLCs to overcome the oral delivery drawback. They have found the \% encapsulation efficiency varies between $76 \pm 12.4 \%$ and $96.6 \pm 7.1 \%$. The high \% of liquid lipid (Capryol ${ }^{\circledR}$ PGMC) causes massive crystal order defects, which lead to more space to entrap the drug molecules and improve the \% EE [44].

4.3.3. Structural Analysis by TEM. The TEM image shown in Figure 10 demonstrated that the RBO-NLCs particles were uniformly distributed with no sign of any aggregation of particles, and the shape of each nanoparticle was spherical. The result indicated that the NLCs particle was dispersed homogenously. A similar outcome was evaluated by Alam and coworkers where the NLCs particles were uniform and in spherical shape [45].

4.4. Powder X-Ray Diffraction (PXRD) Pattern Study. RBO was found to be crystalline in nature as it showed sharp, intense peaks located at 10.0, 14.8, 15.2, 15.7, 20.1, 22.6, and $23.4 \pm 0.2^{\circ} ; 2$ thetas in the powder X-ray diffraction spectrum are shown in Figure 11(a) [46]. The prepared RBO-NLCs were lyophilized to get the powder and used for $\mathrm{p}$-XRD studies to see drug entrapment in the NLCs, as shown in Figure 11(b). The absence of the sharp and intense peaks of the drug in RBO-loaded NLCs indicates that the drug is completely entrapped within the NLCs or the unentrapped drug is converted into the amorphous state [47]. 


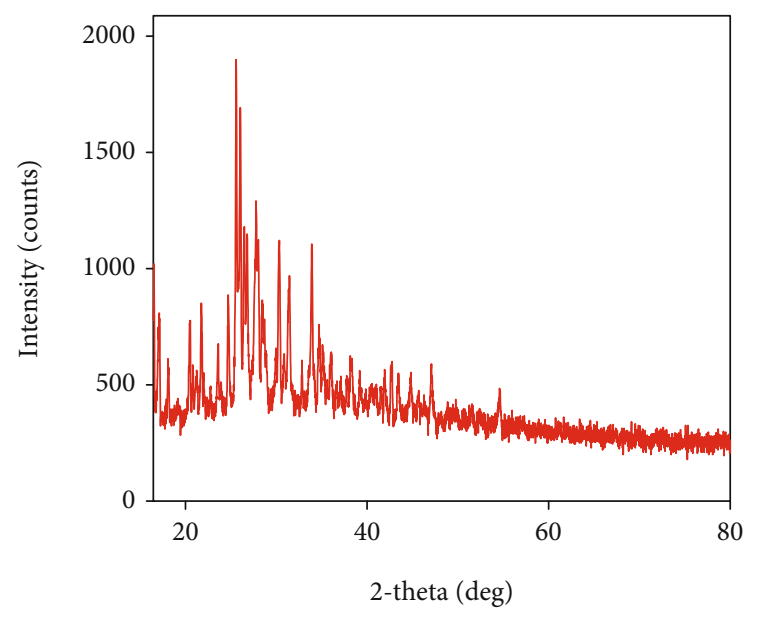

(a)

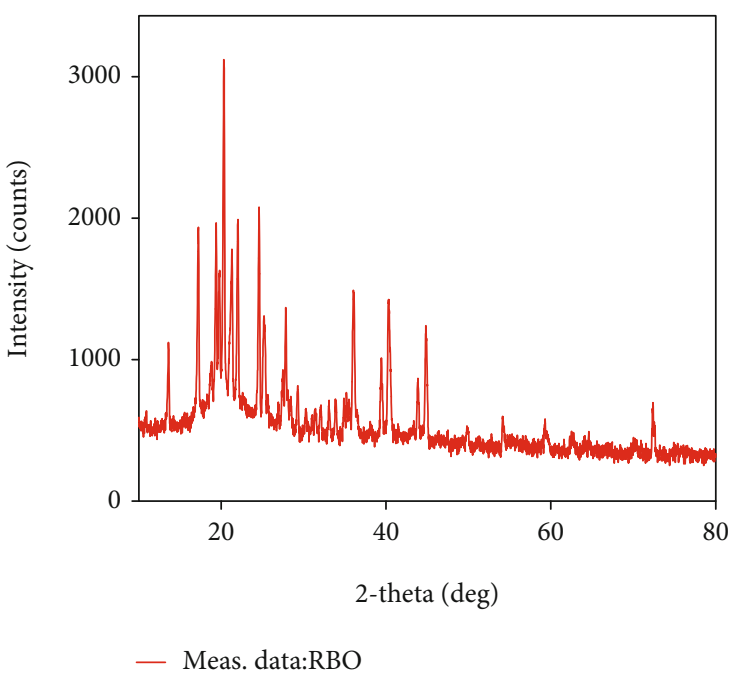

(b)

Figure 11: p-XRD of (a) pure RBO and (b) RBO-NLCs.

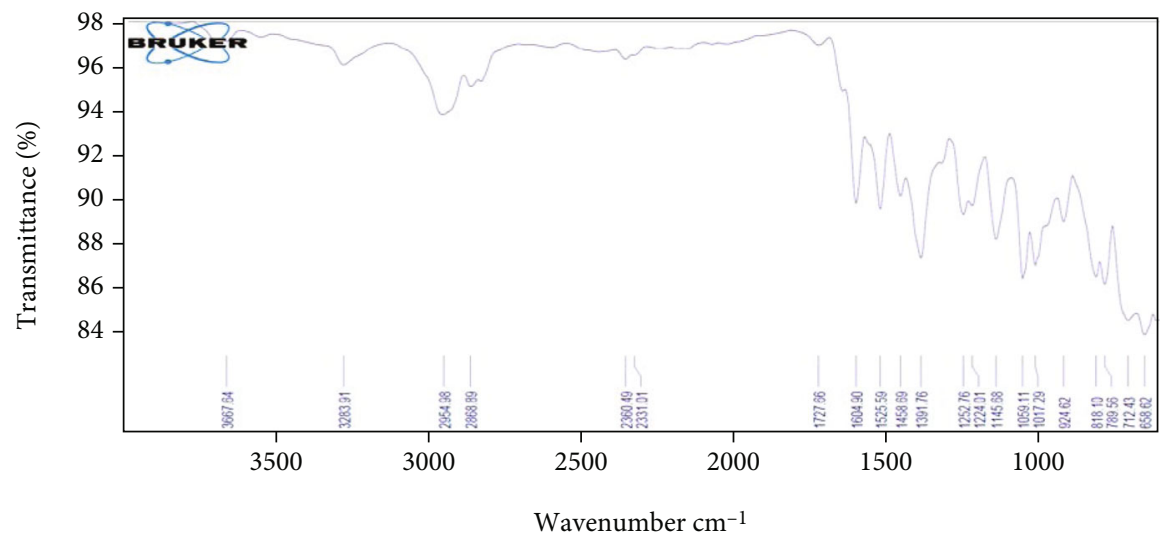

(a)

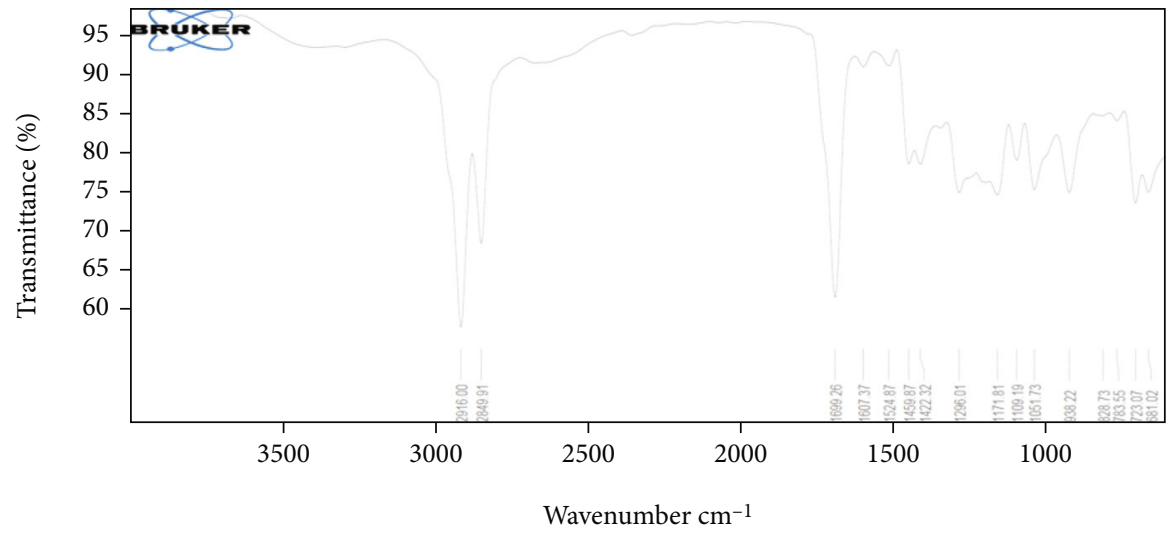

(b)

Figure 12: FT-IR spectra of (a) RBO (drug alone) and (b) RBO-NLCs.

The mannitol used for lyophilization was converted into the amorphous state after freeze-drying and did not show any intense and sharp peaks [48, 49]. Yang and coworkers reported the development of gypenoside-loaded NLCs for oral delivery. The result found that typical peaks of the drug alone were missing in the XRD pattern of formed NLCs, in which attributed drugs in NLCs may appear in an amorphous state [50]. 


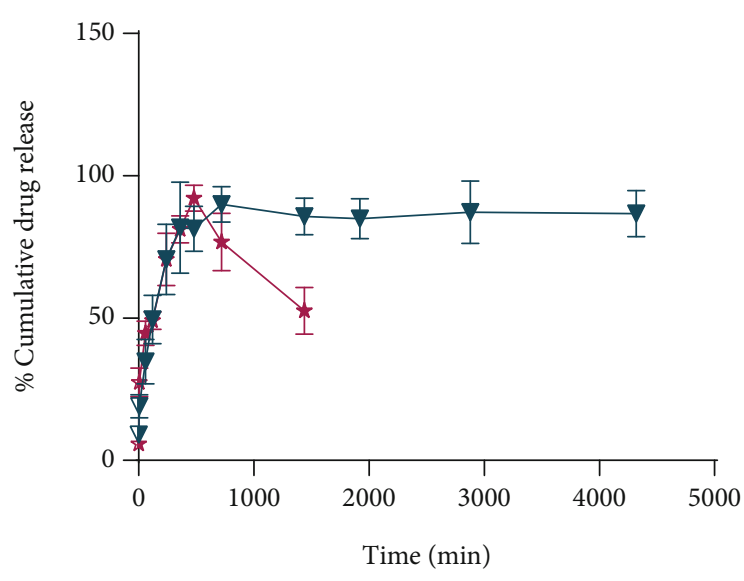

(a)

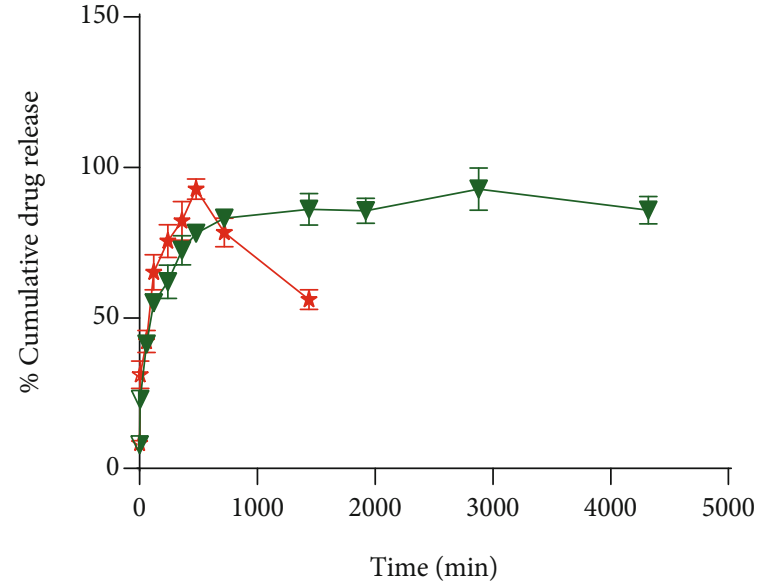

(b)

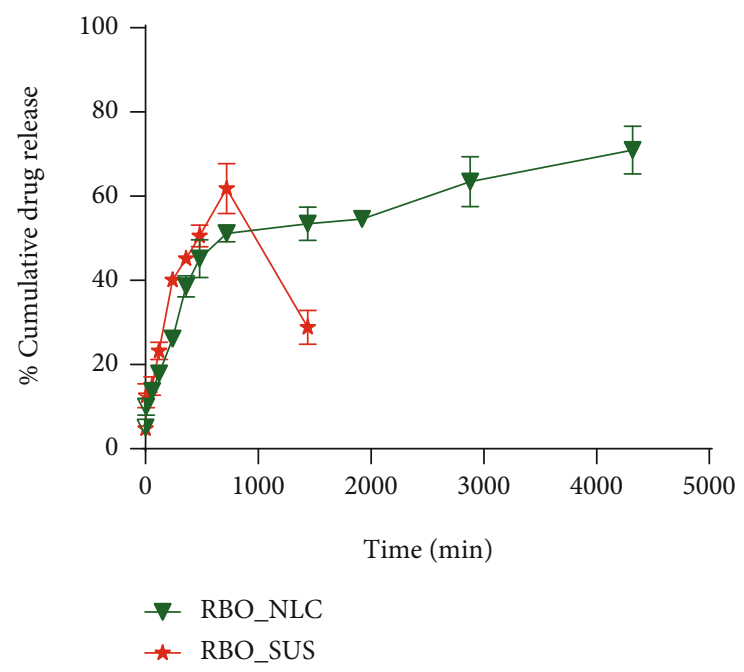

(c)

FiguRE 13: \% CDR of RBO-NLCs and RBO-SUS in various buffers: (a) $0.1 \mathrm{~N} \mathrm{HCl} \mathrm{buffer} \mathrm{pH} \mathrm{1.2;} \mathrm{(b)} \mathrm{acetate} \mathrm{buffer} \mathrm{(AB)} \mathrm{pH} \mathrm{4.5;} \mathrm{(c)} \mathrm{phosphate}$ buffer saline (PBS) pH 6.8.

TABLE 11: Dissolution profile comparison for NLCs and suspension formulation in different dissolution media.

\begin{tabular}{|c|c|c|c|c|}
\hline Parameters & Formulation & 0.1 N HCL (pH 1.2) & $\mathrm{AB}(\mathrm{pH} 4.5)$ & PBS (pH 6.8) \\
\hline \multirow{2}{*}{$\% \mathrm{DE}$} & RBO-NLCs & $83.98 \pm 7.69$ & $84.37 \pm 2.97$ & $55.98 \pm 3.16$ \\
\hline & RBO-SUS & $68.30 \pm 4.64$ & $71.33 \pm 3.06$ & $43.55 \pm 1.48$ \\
\hline f2 similarity factor & - & 43.89 & 43.76 & 48.52 \\
\hline \multirow{2}{*}{$T_{50 \%}(\mathrm{~h})$} & RBO-NLCs & 2.03 & 1.38 & 11.27 \\
\hline & RBO-SUS & 2.05 & 1.20 & - \\
\hline \multirow{2}{*}{$T_{75 \%}(\mathrm{~h})$} & RBO-NLCs & 4.47 & 6.53 & - \\
\hline & RBO-SUS & 4.49 & 3.53 & - \\
\hline
\end{tabular}

4.5. Structural Analysis by FT-IR Spectroscopy. The spectra obtained from FT-IR spectroscopy of drug alone and RBONLCs are demonstrated in Figures 12(a) and 12(b), respectively. The spectra of the drug alone have seen typical peaks at $3667.64 \mathrm{~N}-\mathrm{H}$ stretch, $1727.66 \mathrm{C}=\mathrm{O}$ stretches, $1604.90-$ 1458.69 $\mathrm{C}=\mathrm{C}$ stretches, and 1391.78 C-N stretches. FTIR spectrum of RBO-NLCs showed a peak at 2916 C-H stretch,
2849.51 O-H stretch, and 1699.26 C=O stretch of carboxylic acid, which could be due to the existent of excipients in the NLCs formulation. In the FT-IR spectrum, the typical peak of RBO-NLCs was absent compared to the pure drug because of the complete entrapment of RBO inside the NLCs formulation [51]. Annu et al. have developed the chitosan nanoparticle. The result reported in the FT-IR study stated 
that the characteristic peaks of the drug in nanoparticles were absent compared to drug alone spectra, and it could be the possible reason for entrapment of the drug within the nanoformulation [52].

4.6. In Vitro Drug Release Study. RBO-NLCs and RBO-SUS shown in Figure 13 indicated a sustained drug release at different $\mathrm{pH} 1.2,4.5$, and 6.8 from the initial time point as compared to RBO-SUS, which shows a gradual increase in release but not sustained for $24 \mathrm{~h}$. The drug present inside the lipid matrix of NLCs undergoes surface erosion which is attributed to its sustained release from the formulation. Initial burst release in the NLCs was due to the unentrapped drug. The $t_{75 \%}$ and $t_{50 \%}$ were calculated for each drug formulation. The dissolution profile was compared in terms of \% dissolution efficiency (\%DE) and similarity factor (f2), as shown in Table 11. Results showed a higher \%DE for NLCs formulation in $\mathrm{pH} 1.2,4.5$, and 6.8 compared with RBOSUS.

Similarly, similarity factor, $\mathrm{f} 2$ was calculated. The $\mathrm{f} 2$ values below 50 for $\mathrm{pH} 1.2,4.5$, and 6.8 indicated the both RBO-NLCs and RBO-SUS release pattern were not similar. Sahibzada and coworkers investigated a similar comparison of drug nanoparticle dissolution parameters for the enchantment of oral bioavailability. The \%DE and similarity factor f2 were calculated for two methods to prepare nanoparticles and compared for better dissolution [21].

4.7. Mechanism of Drug Release. The kinetic release model in the buffer of $\mathrm{pH} 1.2,4.5$, and 6.8 was calculated. Based on the $R^{2}$ value, the appropriate kinetic model was chosen for the drug release mechanism from NLCs. So, the KorsmeyerPeppas kinetic release model was selected as the $R^{2}$ value was close to linearity compared to other kinetic release models. This model described release mechanisms from NLCs formulation, which described in three steps the diffusion of water, swelling, and dissolution of the matrix [53]. Duong and coworkers investigated the drug-loaded NLCs, and their drug release kinetic model was compared, and the mechanism was studied using the Korsmeyer-Peppas equation $[18,53,54]$.

4.8. In Vitro Lipolysis Study. The drug should be solubilized near the absorptive membrane so that it is available for systemic absorption. Therefore, the drug should solubilize in an aqueous layer in lipolysis media, and the same will not happen in the case of the sediment layer. The percentage of drug content in a different layer of aqueous and sediment is depicted in Figure 14. The high solubilization potential of the drug in the NLCs formulation in the aqueous layer indicated higher absorption, i.e., approximately $76 \%$ after orally administering the RBO-NLCs formulation. Furthermore, drug content in the sediment phase of RBO-NLCs was also calculated and found to be $18.47 \pm 8.45 \%$. This is because some of the solid lipids might not be digested, so the entrapped drug remains inside the solid lipid. Thus, the result established that RBO-NLCs enhanced its solubilization in the aqueous medium, which simulates the environment of GIT and the intestine. Hence, it can be used as

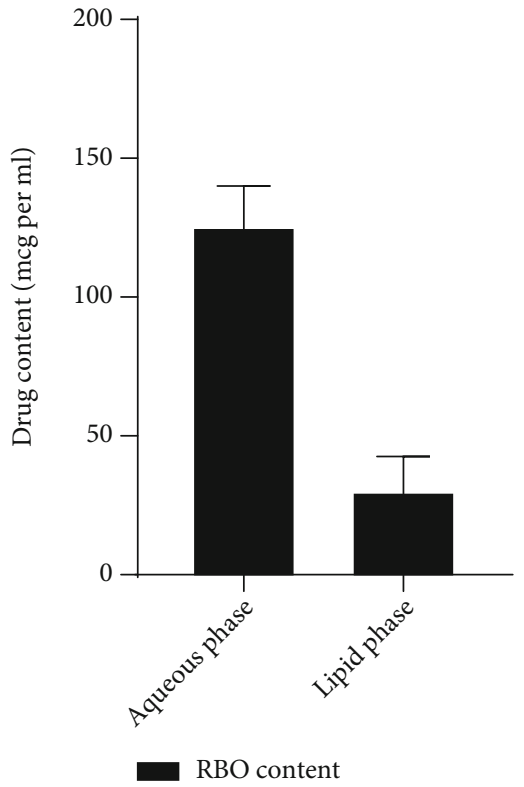

Figure 14: RBO content in different phases of lipolysis media.

in vivo fate of the RBO. A similar investigation was reported by Khan et al. and Rehman et al., in which the drug-loaded NLCs formulation found high drug content available for solubilization in the aqueous phase compared to the sediment phase $[18,55]$.

4.9. In Vitro Haemolysis Study. Haemolysis is detected in red blood cells treated with experimental agents, as shown in Figure 15. The positive control sample showed the least variability in red blood cells with respect to their structure or shape, which was almost similar to the sample treated with NLCs-placebo as the haemolysis count was significantly high in the case of Triton X100 treated sample which destabilizes erythrocytes membrane. The RBO-NLCs formulation showed well intact red blood cells, which were again similar to the NLCs placebo-treated sample (\% haemolysis of $1.855 \%)$. However, slight structure modification occurred in the case of the RBO-SUS sample (\% haemolysis of $2.712 \%)$ treated red blood cells, which disrupts the membrane of red blood cells. The result concluded that the NLCs formulation of RBO was safer compared to a pure drug suspension. So, the result comprises the in vivo fate of NLCs formulation of the selected drug. A similar investigation was reported by Chauhan and associates in which the curcumin nanoformulation evaluated their anticancer potential and evaluated direct nanoformulation erythrocyte interaction in which membrane disruption directly measures the toxicity of nanoformulation [56].

4.10. In Vitro Gut Permeation Study. The intestinal permeability of RBO-NLCs and RBO-SUS is shown in Figure 16. After $2 \mathrm{~h}$ study setup, the flux of the drug in the rat intestine was calculated. The flux for RBO-NLCs and RBO-SUS formulations was found to be $2426.98 \pm 157.77$ and 1241.77 $\pm 68.70 \mu \mathrm{g} / \mathrm{cm}^{2}$, respectively. The apparent permeability coefficient was found to be $75.86 \pm 2.46$ and $38.81 \pm 1.07$ 


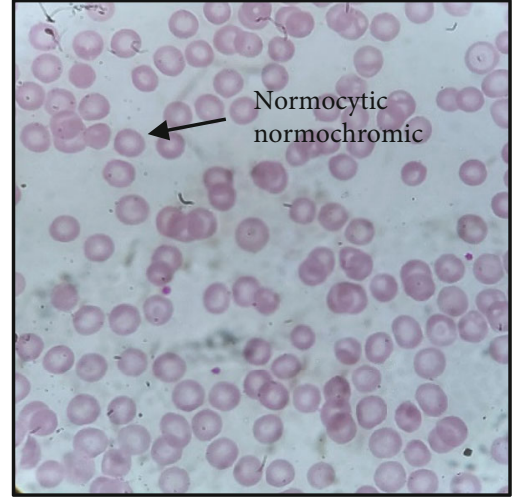

(a) Blank

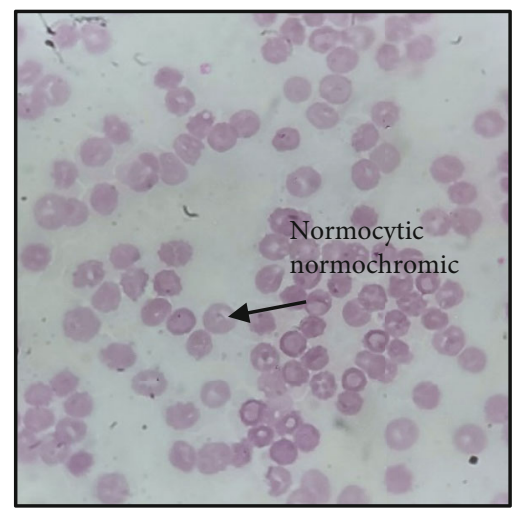

(c) NLCs-placebo

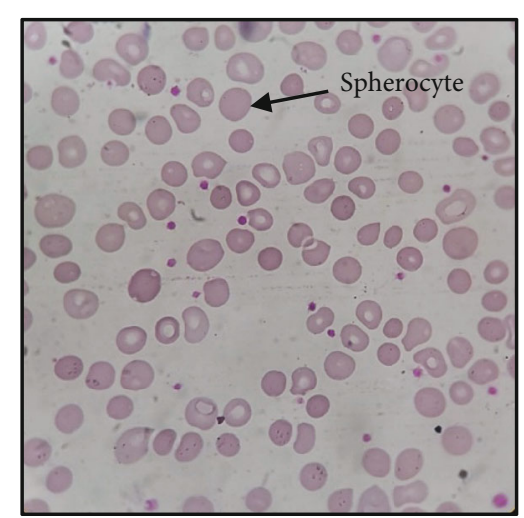

(b) Triton X100

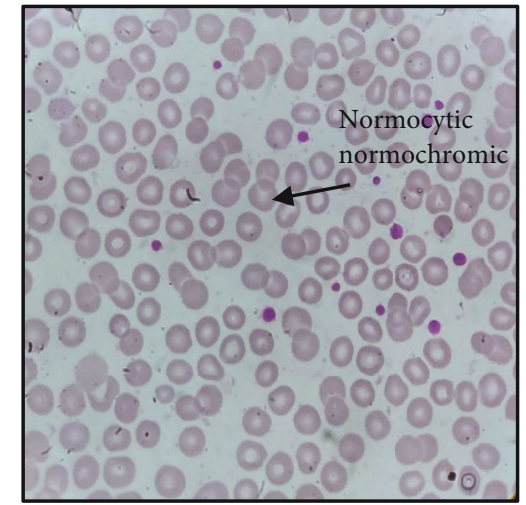

(d) RBO-NLCs

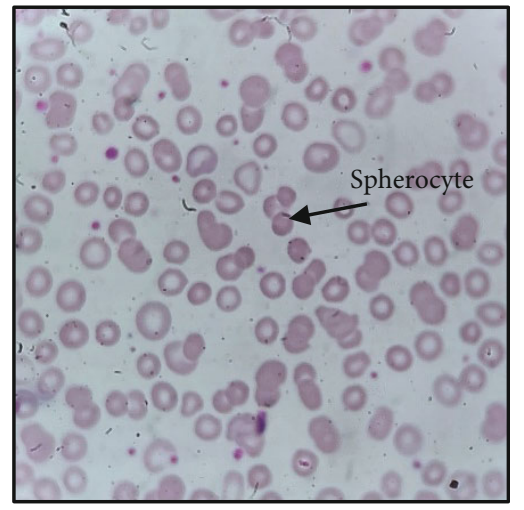

(e) RBO-SUS

FIGURE 15: Haemolysis of red blood cells after being treated with RBO-NLCs formulations and RBO-SUS.

$\mathrm{cm} \min ^{-1}$ for RBO-NLCs and RBO-SUS, respectively. The smaller particle size and permeation enhancer (lipids) of RBO-NLCs enhanced the drug's permeability compared to the RBO-SUS. The reason can be owed to the P-gp inhibitory activity of Solutol HS 15, which inhibits the drug efflux, thereby enhancing the permeability. Almost two times of drug release were observed in RBO-NLCs in contrast to the RBO-SUS formulation [12]. Wu and coworkers investigated the repaglinide-loaded NLCs formulation which demonstrated the membrane permeability compared with their solution. Drug-loaded NLCs with small particle size easily pass the mucus layer and intestinal epithelial cell layer compared with large NLCs [57].
4.11. Depth Permeation Study by Confocal Microscopy. The confocal microscopy images of rat intestine with Rhodamine $\mathrm{B}$ dye, as shown in Figure 17, showed the depth of penetration of RBO-NLCs and RBO-SUS, respectively, after $2 \mathrm{~h}$ of dose administration. The depth of penetration measured through the $z$-axis is $15 \mu \mathrm{m}$ for both formulations. The data obtained distinctly indicated the intensified fluorescence in RBO-NLCs in contrast to RBO-SUS even after $15 \mu \mathrm{m}$ of depth. The result signifies greater penetration of RBO-NLCs, which could be the possible reason for the small particle size of NLCs and the role of lipids and surfactants as penetration enhancers [58]. Soni and coworkers reported the sulforaphane-loaded NLCs, and the result suggested that 


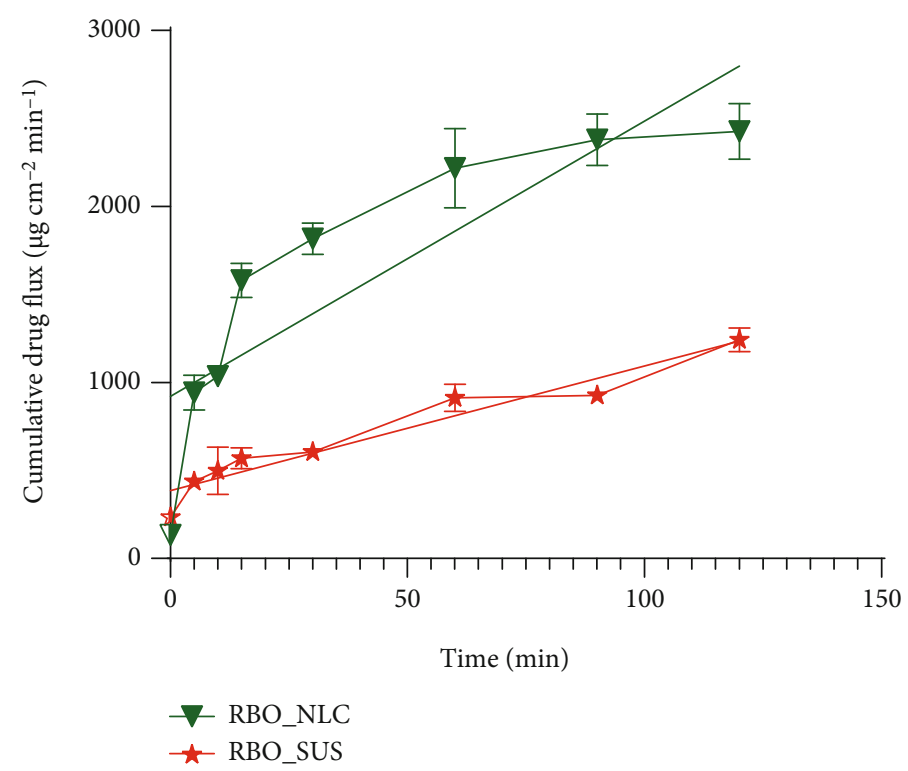

FIgURE 16: The flux of drug permeated in rat intestine at different time point intervals.
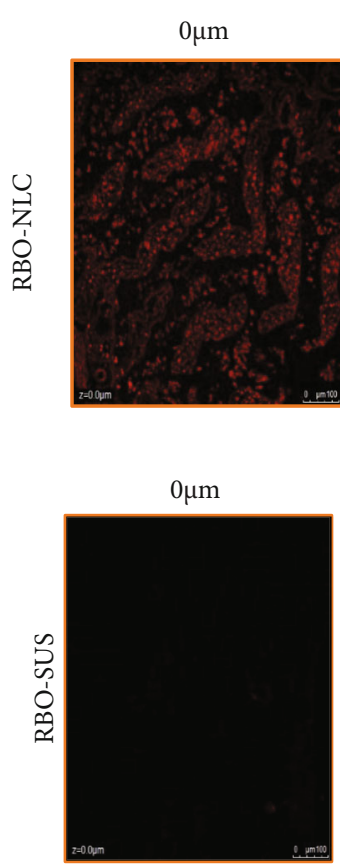

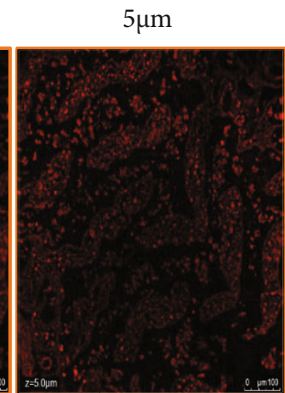

(a)

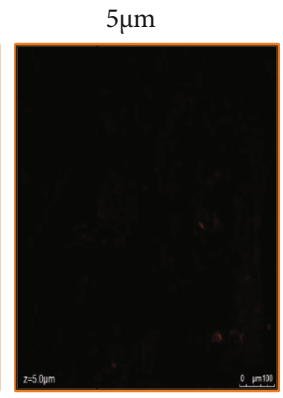

(b)
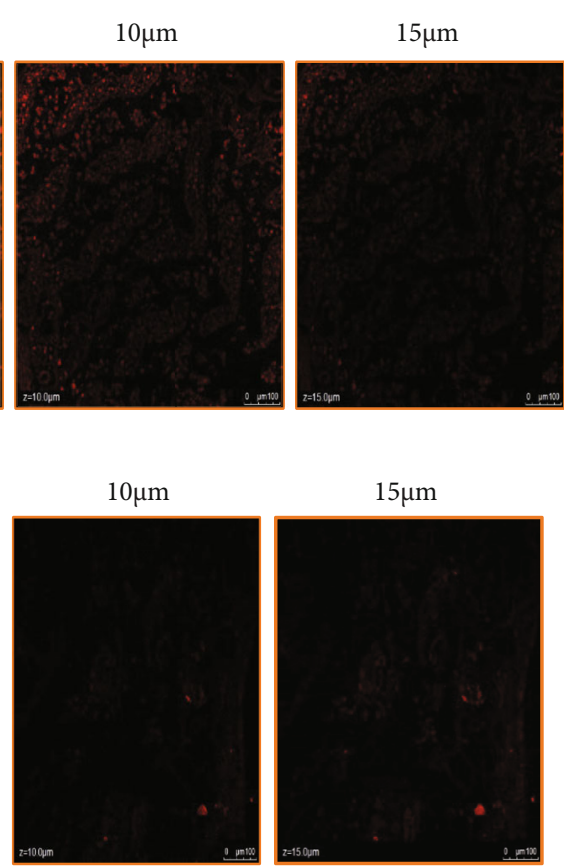

FIGURE 17: Depth of penetration shown in small intestine cells by using CLSM imaging after 2 hours of administration of (a) RBO-NLCs and (b) RBO-SUS.

the depth of permeation of the drug was measured in the intestinal lumen by the depth of fluorescence permeated. Drug-loaded NLCs penetrated more as compared to drug solutions [58].

\subsection{Cell Line Study}

4.12.1. Effect of RBO on MCF-7 Breast Cancer Cells. The antitumor activity of RBO against the MCF-7 cells was investigated by MTT assay. As shown in Figure 18, RBO exhibited a broad spectrum of inhibition against cancer cell lines in a dose-dependent manner. The result from the MTT assay, the $\mathrm{IC}_{50}$ value $(\mu \mathrm{g} / \mathrm{ml})$ of $\mathrm{RBO}$ post- $24 \mathrm{hrs}$ and $48 \mathrm{hrs}$ of treatment against the MCF-7 cell line at $37^{\circ} \mathrm{C}$ at $5 \% \mathrm{CO}_{2}$, was found to be 39.51 and $13.36 \mu \mathrm{g} / \mathrm{ml}$, respectively. After calculation of the $\mathrm{IC}_{50}$ value, similarly dosedependent toxicity of the RBO-NLCs formulation was done as shown in Figure 19. The \% cytotoxicity was significantly enhanced in the case of nanoformulation with lower $\mathrm{IC}_{50}$ value of 0.531 and $0.474 \mu \mathrm{g} / \mathrm{ml}$ post $24 \mathrm{hrs}$ and $48 \mathrm{hrs}$, respectively. So, by formulating into NLCs of the drug, the cytotoxicity was enhanced and reduced their $\mathrm{IC}_{50}$ value by 


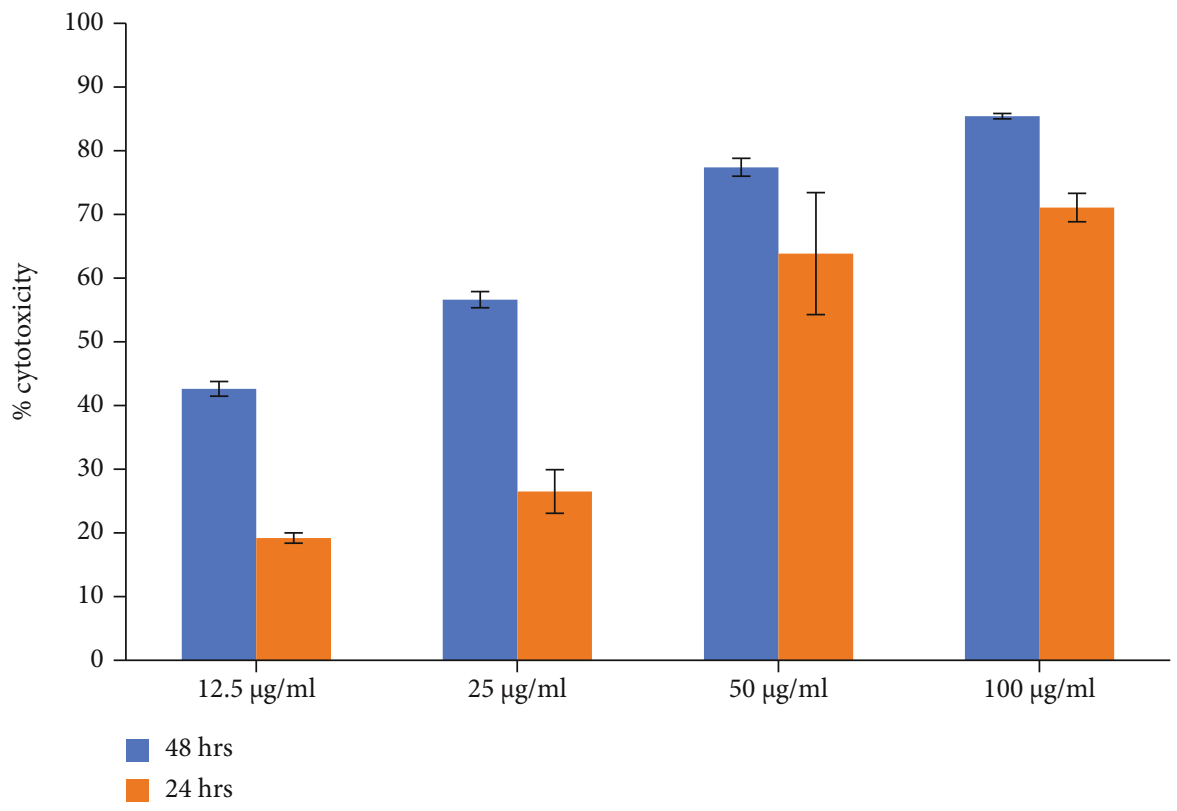

FIGURE 18: Dose-dependent cytotoxicity assay of RBO on MCF-7 cell line at 24 hrs and 48 hrs by MTT.

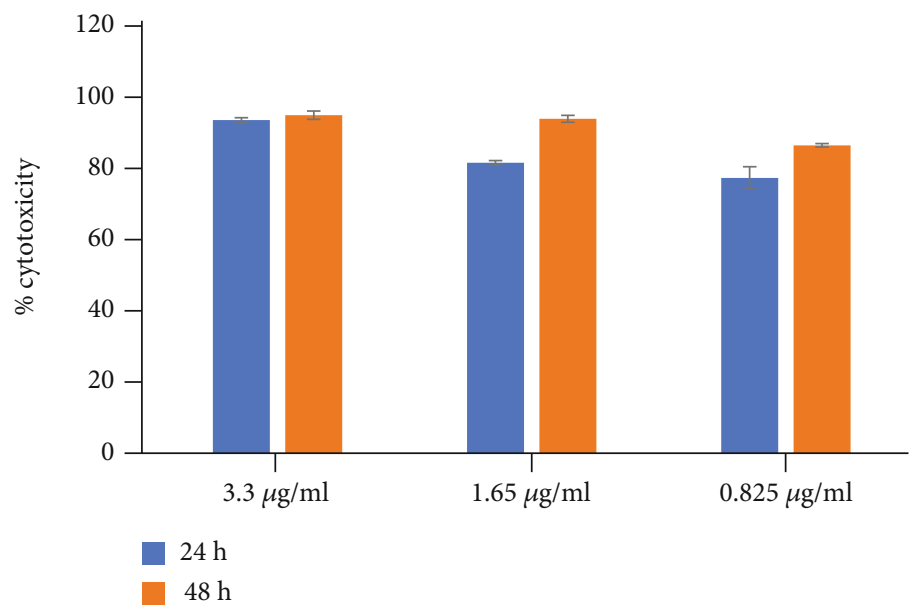

Figure 19: Dose-dependent cytotoxicity assay of RBO-NLCs on MCF-7 cell line at $24 \mathrm{hrs}$ and 48 hrs by MTT.

74 times and 28 times after $24 \mathrm{~h}$ and $48 \mathrm{~h}$, respectively. A similar study was also conducted by Marinelli and coworkers in which RBO alone or in combination with Everolimus was investigated in breast cancer lines [59].

4.13. In Vivo Pharmacokinetic Study. In vivo pharmacokinetic study was conducted in female Wistar rats for the determination of bioavailability data, as depicted into Figure 20 after the oral administration of RBO suspension and RBO-loaded NLCs. The peak plasma concentration $\left(C_{\max }\right)$ and time of occurrence of the same $\left(T_{\max }\right)$ were calculated directly from the drug plasma concentration versus time profile graph. The significant parameters are provided in Table 12 calculated by the linear trapezoidal method using the PK solver add-in tools in Microsoft excel. RBO belongs to the BCS IV drug category which makes it essential to develop a novel drug delivery system so that NLCs can overcome solubility as well the permeability issue. The comparative data of RBO suspension and RBO-NLCs are summarized in Table 12 where $C_{\max }$ and $T_{\max }$ of RBO suspension and RBO-NLCs were found to be $311.90 \pm 36.70$ $\mathrm{ng} / \mathrm{ml}$ and $4.00 \mathrm{~h}$ and $897.92 \pm 28.14 \mathrm{ng} / \mathrm{ml}$ and $4.00 \mathrm{~h}$, respectively. The higher $C_{\max }$ of RBO-NLCs indicated the higher absorption due to their nanosize and lipophilic characteristic of NLCs which is also responsible for micellar solubilization which enhances the lymphatic uptake through Peyer's patches present in the small intestine [60, 61]. The higher $T_{\max }$ also suggested the prolonged and sustained drug release. The in vivo result corroborated with in vitro drug release of $\mathrm{RBO}$ in terms of sustained and longer period of drug release. Higher absorption is achieved due to more of the drug being entrapped inside the lipidic nanoparticles, 


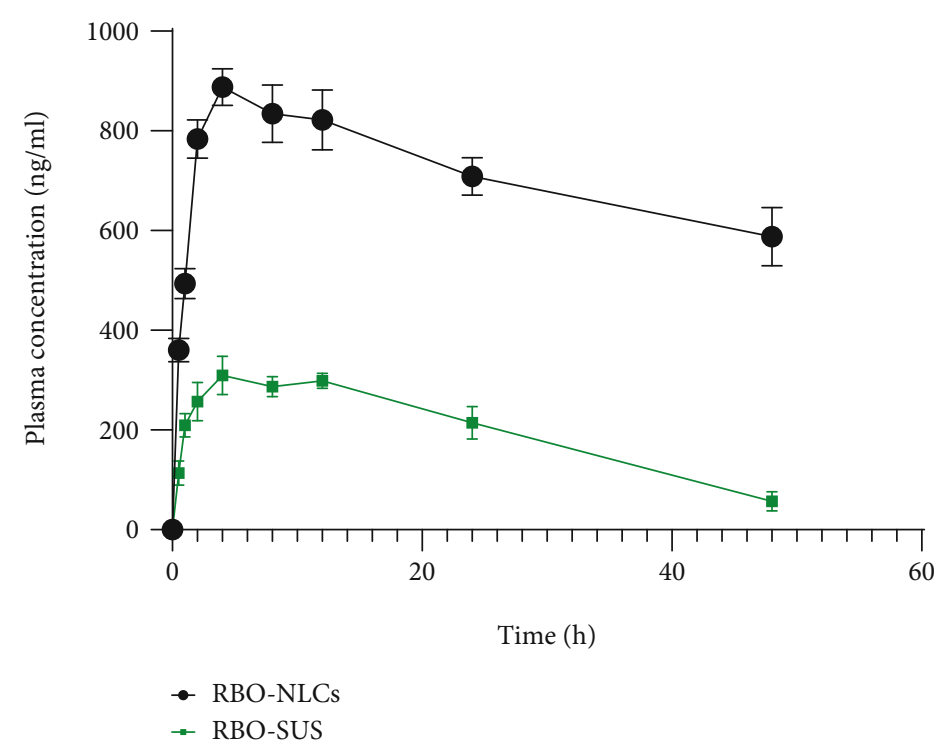

FIGURE 20: The plasma concentration versus time profile curve of RBO-SUS and RBO-NLCs after oral administration. Data expressed in mean \pm standard deviation $(n=3)$.

TABLE 12: Pharmacokinetic parameters of RBO-SUS and RBO-NLCs.

\begin{tabular}{lcc}
\hline Pharmacokinetic parameters & RBO-SUS & RBO-NLCs \\
\hline$C_{\max }(\mathrm{ng} / \mathrm{mL})$ & $311.90 \pm 36.70^{*}$ & $897.92 \pm 28.14^{*}$ \\
$T_{\max }(\mathrm{h})$ & 4.00 & 4.00 \\
$\mathrm{AUC}_{0-t}(\mathrm{ng} \mathrm{h} / \mathrm{mL})$ & $9620.75 \pm 781.21^{*}$ & $34117.67 \pm 548.20^{*}$ \\
$\mathrm{AUC}_{0-\alpha}(\mathrm{ng} \mathrm{h} / \mathrm{mL})$ & $10924.97 \pm 751.39^{*}$ & $94903.96 \pm 21646.64^{*}$ \\
$t_{1 / 2}(\mathrm{~h})$ & $14.95 \pm 3.85$ & $70.53 \pm 18.82$ \\
\hline
\end{tabular}

Data represented as mean \pm standard deviation $(n=3)$ with statistical difference at ${ }^{*} \mathrm{p}<0.001$.

TABLE 13: Result of stability study for RBO-NLCs at $25 \pm 2^{\circ} \mathrm{C} / 60 \pm 5 \% \mathrm{RH}(n=3)$.

\begin{tabular}{lcccccccc}
\hline Time (month) & Phase separation & Sedimentation & Particle size & & PDI & \multicolumn{2}{c}{$\begin{array}{c}\text { Entrapment } \\
\text { efficiency }\end{array}$} \\
\hline 0 & No & No & nm & SD & PDI & SD & $\%$ EE & SD \\
& & & 114.23 & 2.75 & 0.649 & 0.043 & 87.75 & 1.79 \\
1 & No & No & 127.78 & 1.26 & 0.711 & 0.067 & 86.47 & 0.91 \\
3 & No & No & 131.91 & 2.50 & 0.703 & 0.054 & 85.87 & 0.79 \\
6 & No & No & 135.44 & 1.31 & 0.707 & 0.067 & 85.13 & 0.91 \\
\hline
\end{tabular}

and again, the micellar solubilization leads to conversion of nanoparticles into chylomicrons which enhance the lymphatic uptake and hence bypass the hepatic first pass metabolism. From the calculated data, a 3.54-fold increase in the bioavailability of RBO-NLCs was observed as compared to the RBO suspension. Data showed statistical difference at ${ }^{*} p<0.001$ between RBO-SUS and RBO-NLCs.

4.14. Stability Study. The parameters of optimized NLCs on the stability study were evaluated as per the ICH guidelines, and change in any physical and chemical was evaluated as shown in Table 13. A freshly prepared sample was kept for stability study after determining their particle size, and
PDI, which was $114.23 \pm 2.75 \mathrm{~nm}$ and $0.649 \pm 0.043$, respectively, and \% entrapment efficiency was $87.75 \pm 1.79 \%$ on 0 days. As the time point from 1 month to 6 months of stability, the particle size increases from 114.23 to $135.44 \mathrm{~nm}$, as shown in Figure 21(a). It might be because of the swelling of NLCs nanoparticles upon a long period of storage. The entrapment efficiency decreases during the storage time, as shown in Figure 21(b). It might be because solid and liquid lipid integrity decreases and swelling of nanoparticles causes the leakage of the drug, reflecting the decrease in entrapment efficiency. Thus, the drug-loaded NLCs showed good physical and chemical stability upon long storage [45]. The changes in particle size and entrapment efficiency are shown 


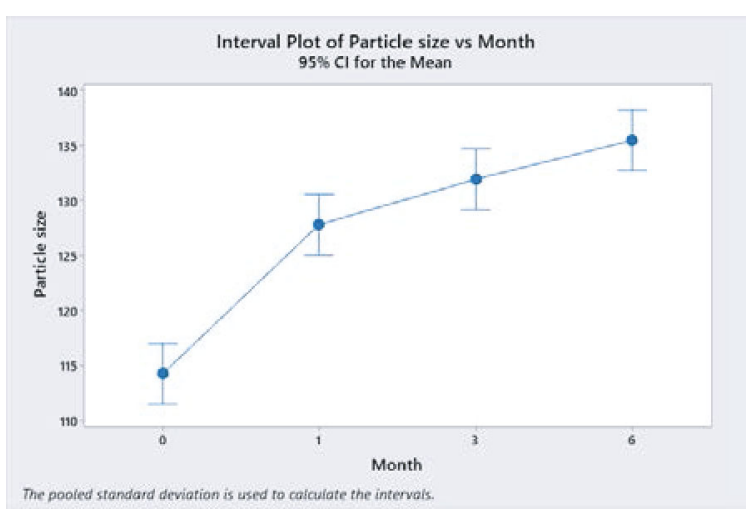

(a)

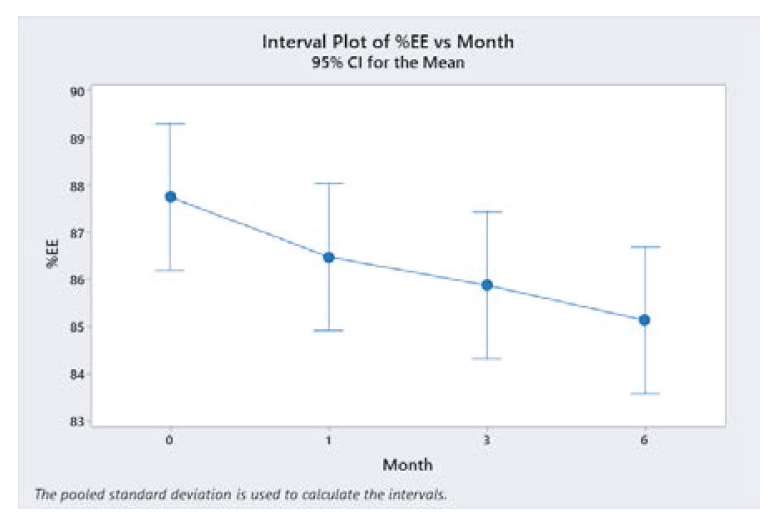

(b)

FIgURe 21: Change in (a) particle size and (b) \% entrapment efficiency upon stability study for 6 months.

in Figures 21(a) and 21(b), respectively, have drawn by using software Minitab 19, Philadelphia, USA. All data obtained from stability corresponded to the preceding research done by Nabi and associates in which they have formulated NLCs using the BBD and stability study done for 90 days [29]

\section{Conclusion}

RBO loaded NLCs was prepared using solvent evaporation followed by the probe sonication method. The NLCs formulation was optimized using the Box-Behnken design response surface method. The prepared NLCs exhibited mean particle size of $114.23 \pm 2.75 \mathrm{~nm}$, mean polydispersity index of $0.649 \pm 0.043$, and high entrapment efficiency of $87.7 \pm 1.79 \%$. TEM images also suggested uniform size and distribution. The in vitro drug release from the NLCs nanoformulation shows a sustained release for a long time (up to $72 \mathrm{~h}$ ), unlike drug suspension, which is only up to $24 \mathrm{~h}$ of drug release, and it could not maintain the sustained drug release for a longer period. The in vitro lipolysis study indicated the drug availability after released from lipidencapsulated drug to the absorption site. The in vitro haemolysis study suggested that the NLCs formulation of the given drug was safe compared to the pure drug. The in vitro permeation study showed an increase in depth of the NLCs formulation due to the lipid used in the formulation, which enhances the permeation as compared to a drug suspension. The cell lines in the MCF-7 study which significantly reduced the dose in the case of the NLCs formulation required to produce similar cytotoxicity when pure drug was used. The findings of the pharmacokinetics study by the in vivo study in female Wistar rats of the RBO-NLCs were carried out which enhance its bioavailability by 3.54 times as compared to the RBO-SUS.

\section{Abbreviations}

$\begin{array}{ll}\text { RBO: } & \text { Ribociclib } \\ \text { NLCs: } & \text { Nanostructured lipid carriers } \\ \text { P-gp: } & \text { P-Glycoprotein } \\ \text { BBD: } & \text { Box-Behnken design }\end{array}$

PDI: Polydispersity index

EE: Entrapment efficiency

TEM: Transmission electron microscopy

$R^{2}$ : $\quad$ Regression value

CDK: Cyclin-dependent kinase

IUPAC: International Union of Pure and Applied Chemistry

HLB: Hydrophilic and lipophilic balance

rcf: Relative centrifugal force

UV: Ultraviolet

QbD: Quality by design

p-XRD: Powder X-ray diffraction

FT-IR: Fourier-transform infrared spectroscopy.

\section{Data Availability}

All data are provided in full in the Results and Discussion section of this paper.

\section{Conflicts of Interest}

All authors declare no conflict of interest.

\section{Acknowledgments}

The authors would like to acknowledge the Indian Council of Medical Research, Govt. of India, New Delhi, India, for providing ICMR-SRF Research fellow to the first author (Grant Number: 45/36/2019-NAN-BMS).

\section{References}

[1] M. R. Ataollahi, J. Sharifi, M. R. Paknahad, and A. Paknahad, "Breast cancer and associated factors: a review," Journal Of Medicine and Life, vol. 8, pp. 6-11, 2015.

[2] S. Malvia, S. A. Bagadi, U. S. Dubey, and S. Saxena, "Epidemiology of Breast Cancer in Indian Women," Asia-Pacific Journal of Clinical Oncology, vol. 13, no. 4, pp. 289-295, 2017.

[3] E. Hamilton and J. R. Infante, "Targeting CDK4/6 in patients with cancer," Cancer Treatment Reviews, vol. 45, pp. 129138, 2016. 
[4] G. N. Hortobagyi, S. M. Stemmer, H. A. Burris et al., "Ribociclib as first-line therapy for HR-positive, advanced breast cancer," The New England Journal Of Medicine, vol. 375, no. 18, pp. 1738-1748, 2016.

[5] J. I. Lai, Y. J. Tseng, M. H. Chen, C. Y. F. Huang, and P. M. H. Chang, "Clinical perspective of FDA approved drugs with Pglycoprotein inhibition activities for potential cancer therapeutics," 2020, http://www.frontiersin.org.

[6] "Center for drug evaluation and research," https://www .accessdata.fda.gov/drugsatfda_docs/nda/2017/ 209092Orig1s000ChemR.pdf.

[7] Z. Fei and M. Yoosefian, "Design and development of polymeric micelles as nanocarriers for anti-cancer ribociclib drug," Journal of Molecular Liquids, vol. 329, article 115574, 2021.

[8] L. Brannon-Peppas and J. O. Blanchette, "Nanoparticle and targeted systems for cancer therapy," 2004, https://pubmed .ncbi.nlm.nih.gov/15350294/.

[9] N. Naseri, H. Valizadeh, and P. Zakeri-Milani, "Solid lipid nanoparticles and nanostructured lipid carriers: structure preparation and application," Advanced Pharmaceutical Bulletin, vol. 5, no. 3, pp. 305-313, 2015.

[10] S. Khan, S. Baboota, J. Ali, S. Khan, R. S. Narang, and J. K. Narang, "Nanostructured lipid carriers: an emerging platform for improving oral bioavailability of lipophilic drugs," International Journal of Pharmaceutical Investigation, vol. 5, no. 4, pp. 182-191, 2015.

[11] S. Khan, M. Shaharyar, M. Fazil, S. Baboota, and J. Ali, "Tacrolimus-loaded nanostructured lipid carriers for oral delivery Optimization of production and characterization," European Journal of Pharmaceutics and Biopharmaceutics, vol. 108, pp. 277-288, 2016.

[12] T. Alam, S. Khan, B. Gaba, M. F. Haider, S. Baboota, and J. Ali, "Adaptation of Quality by Design-Based Development of Isradipine Nanostructured-Lipid Carrier and Its Evaluation for In Vitro Gut Permeation and In Vivo Solubilization Fate," Journal of Pharmaceutical Sciences, vol. 107, no. 11, pp. 2914-2926, 2018.

[13] B. Iqbal, J. Ali, and S. Baboota, "Silymarin loaded nanostructured lipid carrier: from design and dermatokinetic study to mechanistic analysis of epidermal drug deposition enhancement," Journal of Molecular Liquids, vol. 255, pp. 513-529, 2018.

[14] I. Jazuli, Annu, B. Nabi et al., "Optimization of nanostructured lipid carriers of lurasidone hydrochloride using box-behnken design for brain targeting: in vitro and in vivo studies," Journal of Pharmaceutical Sciences, vol. 108, no. 9, pp. 3082-3090, 2019.

[15] A. A. Khan, J. Mudassir, S. Akhtar, V. Murugaiyah, and Y. Darwis, "Freeze-dried lopinavir-loaded nanostructured lipid carriers for enhanced cellular uptake and bioavailability: statistical optimization, in vitro and in vivo evaluations," Pharmaceutics, vol. 11, no. 2, p. 97, 2019.

[16] E. González-Mira, S. Nikolić, M. L. García, M. A. Egea, E. B. Souto, and A. C. Calpena, "Potential use of nanostructured lipid carriers for topical delivery of flurbiprofen," Journal of Pharmaceutical Sciences, vol. 100, no. 1, pp. 242-251, 2011.

[17] Z. Zhang, F. Gao, H. Bu, J. Xiao, and Y. Li, "Solid lipid nanoparticles loading candesartan cilexetil enhance oral bioavailability: in vitro characteristics and absorption mechanism in rats," Nanomedicine: Nanotechnology, Biology, and Medicine, vol. 8, no. 5, pp. 740-747, 2012.
[18] S. Khan, M. Shaharyar, M. Fazil, M. Q. Hassan, S. Baboota, and J. Ali, "Tacrolimus-loaded nanostructured lipid carriers for oral delivery-in vivo bioavailability enhancement," European Journal of Pharmaceutics and Biopharmaceutics, vol. 109, pp. 149-157, 2016.

[19] Q. Luo, J. Zhao, X. Zhang, and W. Pan, "Nanostructured lipid carrier (NLCs) coated with chitosan oligosaccharides and its potential use in ocular drug delivery system," International Journal of Pharmaceutics, vol. 403, no. 1-2, pp. 185-191, 2011.

[20] A. Innes, A. M. Farrell, R. P. Burden, A. G. Morgan, and R. J. Powell, "Complement activation by cellulosic dialysis membranes," Journal of Clinical Pathology, vol. 47, no. 2, pp. 155158, 1994.

[21] M. U. K. Sahibzada, A. Sadiq, S. Khan et al., "Fabrication, characterization and in vitro evaluation of silibinin nanoparticles: an attempt to enhance its oral bioavailability," Drug Design, Development and Therapy, vol. 11, pp. 1453-1464, 2017.

[22] A. C. Salome, C. O. Godswill, and I. O. Ikechukwu, "Kinetics and mechanisms of drug release from swellable and non swellable matrices: a review," Research Journal of Pharmaceutical, Biological and Chemical Sciences, vol. 4, no. 2, pp. 97-103, 2013.

[23] Y. Liu, L. Wang, Y. Zhao et al., "Nanostructured lipid carriers versus microemulsions for delivery of the poorly water-soluble drug luteolin," International Journal of Pharmaceutics, vol. 476, no. 1, pp. 169-177, 2014.

[24] M. M. Cruz Silva, V. M. C. Madeira, L. M. Almeida, and J. B. A. Custódio, "Hemolysis of human erythrocytes induced by tamoxifen is related to disruption of membrane structure," Biochimica et Biophysica Acta (BBA)-Biomembranes, vol. 1464, no. 1, pp. 49-61, 2000.

[25] Z. Attari, A. Bhandari, P. C. Jagadish, and S. Lewis, "Enhanced ex vivo intestinal absorption of olmesartan medoxomil nanosuspension: Preparation by combinative technology," Saudi Pharmaceutical Journal, vol. 24, no. 1, pp. 57-63, 2016.

[26] A. Singh, Y. R. Neupane, B. Mangla, and K. Kohli, "Nanostructured lipid carriers for oral bioavailability enhancement of exemestane: formulation design, in vitro, ex vivo, and in vivo Studies," Journal of Pharmaceutical Sciences, vol. 108, no. 10, pp. 3382-3395, 2019.

[27] B. V. Jardim-Perassi, A. S. Arbab, L. C. Ferreira et al., "Effect of melatonin on tumor growth and angiogenesis in xenograft model of breast cancer," PLoS One, vol. 9, no. 1, article e85311, 2014.

[28] S. Reagan-Shaw, M. Nihal, and N. Ahmad, "Dose translation from animal to human studies revisited," The FASEB Journal, vol. 22, no. 3, pp. 659-661, 2008.

[29] B. Nabi, S. Rehman, S. Aggarwal, S. Baboota, and J. Ali, "Quality by design adapted chemically engineered lipid architectonics for HIV therapeutics and intervention: contriving of formulation, appraising the in vitro parameters and in vivo solubilization potential," AAPS PharmSciTech, vol. 21, no. 7, p. 261, 2020.

[30] K. H. Bang, Y. G. Na, H. W. Huh et al., “The delivery strategy of paclitaxel nanostructured lipid carrier coated with platelet membrane," Cancers, vol. 11, no. 6, p. 807, 2019.

[31] L. M. Negi, M. Jaggi, and S. Talegaonkar, "Development of protocol for screening the formulation components and the assessment of common quality problems of nano-structured lipid carriers," International Journal of Pharmaceutics, vol. 461, no. 1-2, pp. 403-410, 2014. 
[32] C. Zhang, F. Peng, W. Liu et al., "Nanostructured lipid carriers as a novel oral delivery system for triptolide: induced changes in pharmacokinetics profile associated with reduced toxicity in male rats," International Journal of Nanomedicine, vol. 9, no. 1, pp. 1049-1063, 2014.

[33] Z. Teng, M. Yu, Y. Ding et al., "Preparation and characterization of nimodipine-loaded nanostructured lipid systems for enhanced solubility and bioavailability," International Journal of Nanomedicine, vol. 14, pp. 119-133, 2019.

[34] M. Danaei, M. Dehghankhold, S. Ataei et al., "Impact of particle size and polydispersity index on the clinical applications of lipidic nanocarrier systems," Pharmaceutics, vol. 10, no. 2, p. 57, 2018.

[35] S. Cunha, C. P. Costa, J. N. Moreira, J. M. Sousa Lobo, and A. C. Silva, "Using the quality by design (QbD) approach to optimize formulations of lipid nanoparticles and nanoemulsions: a review," Nanomedicine: Nanotechnology, Biology and Medicine, vol. 28, article 102206, 2020.

[36] N. K. Garg, G. Sharma, B. Singh et al., "Quality by Design (QbD)-enabled development of aceclofenac loaded-nano structured lipid carriers (NLCs): An improved dermatokinetic profile for inflammatory disorder(s)," International Journal of Pharmaceutics, vol. 517, no. 1-2, pp. 413-431, 2017.

[37] A. Kovács, S. Berkó, E. Csányi, and I. Csóka, “Development of nanostructured lipid carriers containing salicyclic acid for dermal use based on the quality by design method," European Journal of Pharmaceutical Sciences, vol. 99, pp. 246257, 2017.

[38] P. Pathak and M. Nagarsenker, "Formulation and evaluation of lidocaine lipid nanosystems for dermal delivery," AAPS PharmSciTech, vol. 10, no. 3, pp. 985-992, 2009.

[39] R. K. Subedi, K. W. Kang, and H. K. Choi, "Preparation and characterization of solid lipid nanoparticles loaded with doxorubicin," European Journal of Pharmaceutical Sciences, vol. 37, no. 3-4, pp. 508-513, 2009.

[40] R. Shtay, C. P. Tan, and K. Schwarz, "Development and characterization of solid lipid nanoparticles (SLNs) made of cocoa butter: a factorial design study," Journal of Food Engineering, vol. 231, pp. 30-41, 2018.

[41] M. Noroozi, S. Radiman, and A. Zakaria, "Influence of sonication on the stability and thermal properties of $\mathrm{Al}_{2} \mathrm{O}_{3}$ nanofluids," Journal of Nanomaterials, vol. 2014, 10 pages, 2014.

[42] A. Ahad, M. Aqil, K. Kohli, Y. Sultana, M. Mujeeb, and A. Ali, "Formulation and optimization of nanotransfersomes using experimental design technique for accentuated transdermal delivery of valsartan," Nanomedicine: Nanotechnology, Biology and Medicine, vol. 8, no. 2, pp. 237-249, 2012.

[43] J. Varshosaz, M. A. Davoudi, and S. Rasoul-Amini, "Docetaxel-loaded nanostructured lipid carriers functionalized with trastuzumab (herceptin) for HER2-positive breast cancer cells," Journal of Liposome Research, vol. 28, no. 4, pp. 285295, 2018.

[44] M. Elmowafy, H. M. Ibrahim, M. A. Ahmed, K. Shalaby, A. Salama, and H. Hefesha, "Atorvastatin-loaded nanostructured lipid carriers (NLCs): strategy to overcome oral delivery drawbacks," Drug Delivery, vol. 24, no. 1, pp. 932-941, 2017.

[45] T. Alam, J. Pandit, D. Vohora, M. Aqil, A. Ali, and Y. Sultana, "Optimization of nanostructured lipid carriers of lamotrigine for brain delivery: in vitro characterization and in vivo efficacy in epilepsy," Expert Opinion on Drug Delivery, vol. 12, no. 2, pp. 181-194, 2015.
[46] Ratiopharm GmbH 89079 Ulm (DE), "Crystalline forms of ribociclib free base," 2017, https://patentimages.storage .googleapis.com/58/4f/69/f2481b0c2868b1/EP3156406A1.pdf.

[47] L. J. Jia, D. R. Zhang, Z. Y. Li et al., "Preparation and characterization of silybin-loaded nanostructured lipid carriers," Drug Delivery, vol. 17, no. 1, pp. 11-18, 2010.

[48] S. Torrado and S. Torrado, "Characterization of physical state of mannitol after freeze-drying: effect of acetylsalicylic acid as a second crystalline cosolute," Chemical \& Pharmaceutical Bulletin, vol. 50, no. 5, pp. 567-570, 2002.

[49] R. K. Cavatur, N. M. Vemuri, A. Pyne, Z. Chrzan, D. ToledoVelasquez, and R. Suryanarayanan, "Crystallization behavior of mannitol in frozen aqueous solutions," Pharmaceutical Research, vol. 19, no. 6, pp. 894-900, 2002.

[50] G. Yang, F. Wu, M. Chen, J. Jin, R. Wang, and Y. Yuan, "Formulation design, characterization, and in vitro and in vivo evaluation of nanostructured lipid carriers containing a bile salt for oral delivery of gypenosides," International Journal of Nanomedicine, vol. Volume 14, pp. 2267-2280, 2019.

[51] B. Shah, D. Khunt, M. Misra, and H. Padh, "Non-invasive intranasal delivery of quetiapine fumarate loaded microemulsion for brain targeting: formulation, physicochemical and pharmacokinetic consideration," European Journal of Pharmaceutical Sciences, vol. 91, pp. 196-207, 2016.

[52] Annu, S. Baboota, and J. Ali, "In vitro appraisals and ex vivo permeation prospect of chitosan nanoparticles designed for schizophrenia to intensify nasal delivery," Polymer Bulletin, pp. 1-23, 2021.

[53] V. A. Duong, T. T. L. Nguyen, H. J. Maeng, and S. C. Chi, "Data on optimization and drug release kinetics of nanostructured lipid carriers containing ondansetron hydrochloride prepared by cold high-pressure homogenization method," Data in Brief, vol. 26, article 104475, 2019.

[54] A. S. Joshi, H. S. Patel, V. S. Belgamwar, A. Agrawal, and A. R. Tekade, "Solid lipid nanoparticles of ondansetron $\mathrm{HCl}$ for intranasal delivery: development, optimization and evaluation," Journal of Materials Science: Materials in Medicine, vol. 23, no. 9, pp. 2163-2175, 2012.

[55] S. Rehman, B. Nabi, S. Baboota, and J. Ali, "Tailoring lipid nanoconstructs for the oral delivery of paliperidone: Formulation, optimization and in vitro evaluation," Chemistry and Physics of Lipids, vol. 234, p. 105005, 2021.

[56] S. Chauhan, M. Yallapu, Ebeling, Chauhan, and Jaggi, "Interaction of curcumin nanoformulations with human plasma proteins and erythrocytes," International Journal of Nanomedicine, vol. 6, p. 2779, 2011.

[57] L. Wu, L. Zhao, X. Su, P. Zhang, and G. Ling, "Repaglinideloaded nanostructured lipid carriers with different particle sizes for improving oral absorption: preparation, characterization, pharmacokinetics, andin situintestinal perfusion," Drug Delivery, vol. 27, no. 1, pp. 400-409, 2020.

[58] K. Soni, M. Rizwanullah, and K. Kohli, "Development and optimization of sulforaphane-loaded nanostructured lipid carriers by the Box-Behnken design for improved oral efficacy against cancer:in vitro,ex vivoandin vivoassessments," Artificial Cells, Nanomedicine, and Biotechnology, vol. 46, no. sup1, pp. 15-31, 2018.

[59] O. Marinelli, E. Romagnoli, F. Maggi et al., "Exploring treatment with ribociclib alone or in sequence/combination with everolimus in ER+HER2-Rb wild-type and knock-down in breast cancer cell lines," BMC Cancer, vol. 20, no. 1, p. 1119, 2020. 
[60] S. Jain, S. R. Patil, N. K. Swarnakar, and A. K. Agrawal, "Oral delivery of doxorubicin using novel polyelectrolyte-stabilized liposomes (layersomes)," Molecular Pharmaceutics, vol. 9, no. 9, pp. 2626-2635, 2012.

[61] A. K. Jain, N. K. Swarnakar, C. Godugu, R. P. Singh, and S. Jain, "The effect of the oral administration of polymeric nanoparticles on the efficacy and toxicity of tamoxifen," Biomaterials, vol. 32, no. 2, pp. 503-515, 2011. 